\title{
Micro/Nanostructures for Far-Field Thermal Emission Control: An Overview
}

\author{
Boxiang Wang, Mengqi Liu, Tiancheng Huang and Changying Zhao
}

Engineering thermal emission using micro/nanostructures has received growing attention in recent years. A variety of novel coherent, directional, polarized and narrowband thermal light sources as well as wavelength selective emitters have been proposed and investigated. These emitters show a great potential in applications like thermophotovoltaics, solar energy concentration, radiative cooling, thermal detection, sensing, thermal camouflage, thermal rectification, etc. In this article, we give a review on the use of micro/nanostructures to control far-field thermal emission. Thermal emitters based on various micro/nanostructures including gratings, photonic crystals, metamaterials and metasurfaces are reviewed and some important applications are summarized. We expect this article can provide a general overview of this field.

Keywords: Thermal radiation; Micro/nanostructures; Thermal emitters; Metamaterials and metasurfaces; Photonic crystals

Received 29 September 2019, Accepted 26 November 2019

DOI: $10.30919 /$ esee 8 c360

\section{Introduction}

Thermal radiation of natural bulk materials is usually incoherent, that is, the emitted electromagnetic waves are usually omnidirectional, unpolarized and constitute a broad spectrum. ${ }^{1}$ These characteristics largely impede the efficient utilization of thermal radiation energy that virtually exist everywhere. On the other hand, in the past over three decades, with the rapid development of micro/nanofabrication technologies, a variety of micro/nanostructures, including gratings, photonic crystals, plasmonic structures, metamaterials and metasurfaces, etc., have been proposed and extensively studied to achieve exotic optical and electromagnetic properties like negative refraction, ${ }^{2}$ reversed Cherenkov radiation, ${ }^{3}$ subdiffraction focusing and imaging, ${ }^{4}$ cloaking, ${ }^{5}$ slow light, ${ }^{6}$ and so on, which, in general, result from the strong lightmatter interactions at micro- and nanoscale. Therefore, these artificial micro/nanostructures provide an unprecedented route to manipulating the characteristic of thermal radiation.

Recently there has been a lot of efforts in engineering thermal radiation using aforementioned optical/electromagnetic micro/nanostructures, ${ }^{7-10}$ and a variety of novel coherent, directive, polarized and narrowband thermal light sources as well as wavelength selective emitters have been designed and manufactured, which have remarkably different thermal emission properties from conventional bulk materials. These novel thermal emitters are quite promising for a plethora of important applications like thermophotovoltaics (TPV), ${ }^{11,12}$ solar energy concentration, ${ }^{13}$ radiative cooling, ${ }^{14,15}$ thermal detection and sensing, ${ }^{16-18}$ novel light sources, ${ }^{19}$ thermal camouflage ${ }^{20,21}$ and thermal rectification, ${ }^{7-9}$ etc. Witnessing the rapid development of this field dubbed thermal photonics in recent years, ${ }^{7}$ in this article, we give an introductory review on the use of

Institute of Engineering Thermophysics, School of Mechanical Engineering, Shanghai Jiao Tong University, Shanghai, 200240, China

*E-mail: changying.zhao@sjtu.edu.cn micro/nanostructures to control thermal emission. Our attention is only paid to far-field emitters, and for near-field thermal radiation control, see the reviews Refs. [22-25]. In particular, we focus on how different types of artificial micro/nanostructures, including gratings, photonic crystals, metamaterials and metasurfaces, can be engineered to achieve desirable thermal emission properties for different applications.

This article is organized as follows. We first review several important types of micro/nanostructures to tailor thermal radiation. More specifically, in Section 2, thermal emitters based on grating structures are discussed. In Section 3, photonic crystal-based thermal emitters are reviewed. In Section 4, we review metamaterial-based thermal emitters, including general MIM-type metamaterials, epsilonnear-zero and hyperbolic metamaterials as well as disordered/aperiodic metamaterials. In Section 5, we give an overview of various metasurface thermal emitters. In Section 6, we pay a special attention to some novel reconfigurable thermal emitters. After introducing these micro/nanostructures, in Section 7, we introduce some typical applications of thermal emitters, including directive, narrowband and/or polarized thermal radiation sources, daytime radiative cooling, thermophotovoltaics and thermal camouflage. In Section 8, a summary along with an outlook is presented.

\section{Emitters based on gratings}

One of the earliest attempts to create artificial emitters is based on the grating structure. A simple one-dimensional (1D) grating is shown in Fig. 1(a), reprinted from Yoon et $a l^{26}$ where $\Lambda$ is the period of the grating, $w$ is the ridge width with a filling ratio defined as $f=w / \Lambda$ and $h$ is the height of the ridge. Theoretical works were conducted in the 1980s and 1990s, e.g., Refs.[27-30]. A pioneering experimental work was presented by Hesketh et $a l^{31}$, who investigated the thermal emission from 1D deep gratings. Normal spectral (3 $\mu \mathrm{m}-14 \mu \mathrm{m})$ emissivity measurements were carried out for $45 \mu \mathrm{m}$ deep, near square-wave gratings made of heavily phosphorus doped silicon at $400{ }^{\circ} \mathrm{C}$ for both $s$ and $p$-polarizations. Different values of the period $\Lambda$ of the grating 
were investigated, including $10,14,18$ and $22 \mu \mathrm{m}$, resulting a $\Lambda / \lambda$ ranging from 0.14 to 7.33 . They showed that all these deep gratings exhibit significantly higher spectral emissivity than the purely smooth surface of doped silicon in the entire investigated spectral range. For both $s$ - and $p$ - polarized emission, they found that the resonances in the spectra are due to the excitation of standing waves in the air slots perpendicular to the silicon surface, very similar to those in an organ pipe. They further revealed that the resonant amplitude of the $s$ polarization does not depend significantly on $\Lambda$, while that of the $p$ polarization does. Greffet $e t a l^{32}$ experimentally demonstrated the highly directional thermal emission from 1D silicon carbide ( $\mathrm{SiC}$ ) gratings. The highly directional coherent thermal emission, stems from the diffraction of surface phonon polaritons (SPhPs) by the grating structure, which can provide a momentum (wave vector) compensation to convert surface waves to propagating waves. More precisely, in this study, the resonant emission angle $\theta$ at an emission wavelength $\lambda$ can be theoretically determined by the following simple 1D grating equation $\operatorname{as}^{32-34}$

$$
k_{\mathrm{SPhP}}=k_{x}+m K_{\mathbf{G} x}
$$

where $m$ is an integer denoting the diffraction order, $k_{x}=2 \pi \sin \theta / \lambda$ is the $x$ - component of the wavevector of emitted waves, $K_{G x}=2 \pi / \Lambda$ is the grating reciprocal vector, and $k_{\mathrm{SPh}}$ is the wavevector of the surface wave that is given by

$$
k_{\mathrm{SPhP}}=k_{0}\left(\frac{\varepsilon_{1}+\varepsilon_{2}}{\varepsilon_{1} \varepsilon_{2}}\right)^{1 / 2}
$$

for $\mathrm{SPhPs}$ propagating along the $x$-direction, where $\varepsilon_{1}$ and $\varepsilon_{2}$ are permittivities of the surrounding medium and $\mathrm{SiC}$ material respectively. Similarly, (quasi-)coherent thermal radiation sources can be realized by combing the simple grating structure and surface plasmon polaritons (SPPs), as in a recent work carried out by Liu et al..$^{35}$ The fabricated 1D grating made of TiN, a refractory plasmonic material, the thermal emissivity spectrum and the angular profile at the wavelength of 3.06 $\mu \mathrm{m}$, are presented in Fig. 1(b), where a highly narrow band and directional thermal emission phenomenon is observed. Chen et $a l^{36}$ also proposed a design for wavelength selective emitters based on complex gratings realized by the superposition of two simple binary 1D gratings made of tungsten with different periodicities. The calculation was done through the rigorous coupled wave analysis (RCWA) algorithm. It was shown the combination of different periodicities enables a wide peak within the wavelength region of interest due to the excitation of SPPs for TM waves. The designed emitters are very suitable as thermophotovoltaic radiators. Note for 1D deep gratings, Fabry-Perotlike microcavity resonances due to the multiple reflections of grating ridges can be also excited and lead to more densely populated optical states, therefore significantly enhancing thermal emission. ${ }^{26}$ The resonance wavelength of microcavity modes $\lambda$ can be theoretically estimated by the following formula for TE waves ${ }^{3740}$ (which are in essence a type of waveguide modes manifested as discrete energy levels)

$$
\frac{2 \pi}{\lambda}=\sqrt{\left(\frac{\pi m}{a}\right)^{2}+\left(\frac{\pi l}{h}\right)^{2}}
$$

where $m$ and $l$ are integers indicating the orders of cavity resonance, and $a=(1-f) \Lambda$ and $h$ are the grating slot width and depth as shown in Fig. 1(a), respectively. Nguyen-Huu et $a l^{40}$ demonstrated that due to the excitation of cavity resonances, the peak position of the emission/absorption spectra of 1D deep gratings heavily depends on the depth of the grooves. This microcavity resonance is usually regarded as an angle-independent effect and therefore very suitable for TPV applications requiring an omnidirectional high emissivity. ${ }^{40}$

Two-dimensional (2D) gratings have also been extensively studied as thermal emitters. A seminal work was conducted by Heinzel et al., (a)

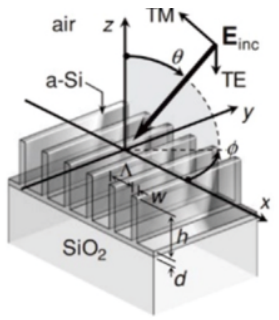

(b)
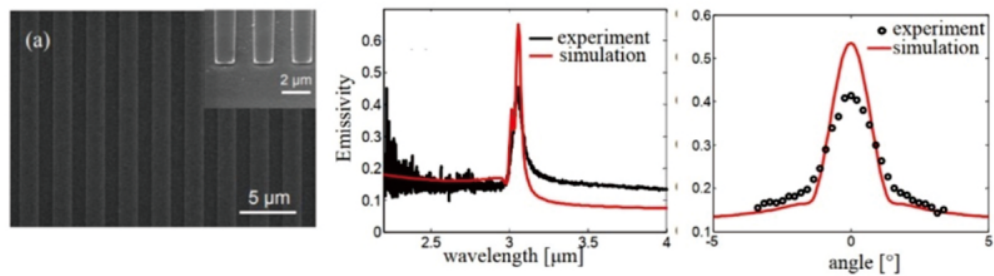

(c)
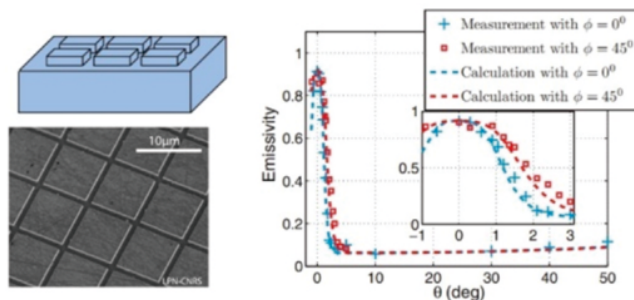

(d)
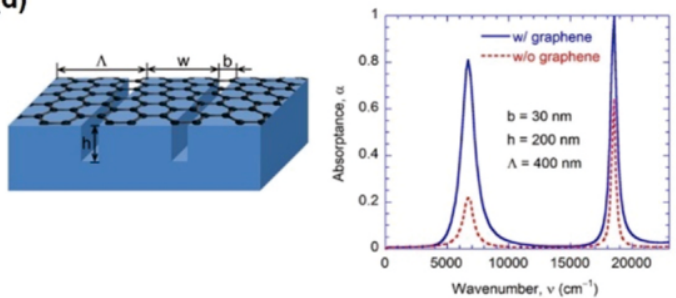

Fig. 1 Thermal emitters based on grating structures. (a) Schematic of 1D simple binary gratings made of amorphous silicon (a-Si). Reprinted with permission from Ref. [26]. Copyright 2014, Wiley-VCH Verlag GmbH \& Co. KGaA, Weinheim. (b) (Left panel) SEM image of the fabricated 1D grating made of TiN after heated up for $32 \mathrm{~h}$ with an inset showing the tilted view. (Middle panel) The measured and simulated emissivity spectrum. (Right panel) The measured and simulated angular profile of emissivity at the wavelength of $3.06 \mu$ m. Reprinted with permission from Ref. [35]. Copyright 2015, Optical Society of America. (c) (Left panel) Schematic of the 2D grating made of SiC, along with the SEM image of fabricated microstructure. (Right panel) The measured and calculated angular profile of thermal emissivity at the wavelength of $12.18 \mu \mathrm{m}$ for two different azimuthal angles $\left(\phi=0^{\circ}\right.$ and $\left.\phi=45^{\circ}\right)$. Reprinted with permission from Ref. [41]. Copyright 2012, American Physical Society. (d) (Left panel) Schematic of the graphene-covered 1D Ag deep grating. (Right panel) The normal absorptivity/emissivity of the graphene-covered (with graphene) and plain (w/o graphene) Ag deep grating. Reprinted with permission from Ref. [42]. Copyright 2014, AIP Publishing. 
which theoretically and experimentally investigated 2D grating made of tungsten was deposited on a dielectric film. The resonant frequencies of the emissivity of the 2D tungsten gratings can be theoretically determined by a combination of the dispersion relation of SPPs and the grating equation like the $1 \mathrm{D}$ case, and the measured spectral emissivity clearly exhibited the designed emission resonances as predicted. This type of radiation emitter exhibited a strong angle dependence of the spectral emissivity, which can also be utilized to achieve coherent thermal sources. For instance, Arnold et $a l^{41}$ experimentally showed that the thermal excitation of surface-phonon polaritons on the surface of 2D grating can result a highly anisotropic thermal light source. The schematic of the 2D grating, the fabricated microstructure and the calculated/measured angular profile of thermal emissivity are given in Fig. 1(c). The measured angular width of emissivity peak is about 3.9 deg. Besides, there are also many works that utilized microcavity resonance modes in deep $2 \mathrm{D}$ gratings to further manipulate thermal radiation, ${ }^{4446}$ to achieve narrowband ${ }^{46}$ or wavelength-selective ${ }^{45}$ emitters, to name a few.

Due to their relative easiness of fabrication, recently, there are still a bunch of works carried out on grating structures to further improve the emission performance and achieve multifunctionalities using modified structures and novel materials. For instance, Song et al. ${ }^{47}$ investigated the thermal emission from a trilayer $\mathrm{W} / \mathrm{SiO}_{2} / \mathrm{W}$ grating structure using the FDTD method. By properly designing the geometric parameters, a high average emissivity of 0.95 is obtained for TM waves in the range around $600-1900 \mathrm{~nm}$, and a broadband emission peak with near-unity emissivity was achieved in the range of 900-1800 nm, which is attributed to the excitation of magnetic polariton (MP) resonances and explained through an analogous inductor-capacitor (LC) circuit model. ${ }^{48,49}$ Note MP resonances result from the strong coupling of electromagnetic waves with the magnetic resonance excited inside the nanostructures. ${ }^{48-53}$ In such 1D grating structure, MPs are manifested as strongly localized magnetic field inside the slit between two neighboring metallic strips, with induced electric current flowing along the metal surface. ${ }^{48,49}$ Graphene and other 2D materials can be added to gratings to further enhance the thermal emission/absorption performance. For example, Zhao et al. ${ }^{42}$ demonstrated that the combination of a graphene layer and a deep 1D Ag grating, schematically shown in the left panel of Fig.1(d), can lead to the absorption/emission enhancement for both owing to the excitation of MPs. The absorption/emission spectrum with and without graphene is presented in the right panel of Fig. 1(d). Recently, grating structures have also been elaborated to realize nonreciprocal emission and absorption. For instance, by applying a magnetic field, Zhao et al. ${ }^{54}$ investigated the thermal emission of a $1 \mathrm{D} \mathrm{SiC}$ grating deposited on a magneto-optical layer made of InAs and demonstrated that this strong magneto-optical response leads to a substantial violation of Kirchhoff's law.

\section{Emitters based on photonic crystals}

Artificial pass bands and forbidden bands (i.e. photonic band gaps, PBGs) can be engineered by the arrangements and periodicity of photonic crystals (PC), which can also serve as an effective tool to tailor thermal emission. In general, the PBGs are generated by the destructive interference of scattered waves from different lattice points, which are described by Bragg's diffraction law. ${ }^{55}$ In this sense, the scale of micro/nanostructures is usually comparable to the operating wavelength. Thermal emitters based on photonic crystals have the potential to realize narrowband, directive, polarized and even tunable emission, which have drawn much attention in the last several decades. According to the dimensions of micro/nanostructures in photonic crystals, we can divide them into three types: one-dimensional (1D), two-dimensional (2D) and three-dimensional (3D) photonic crystal thermal emitters.

Although being the simplest PC thermal emitters, 1D PC thermal emitters can still provide reliable control of thermal emission with an easy fabrication process. As shown in Fig. 2(a), Narayanaswamy and $\mathrm{Chen}^{56}$ presented a one-dimensional photonic crystal made of thin metal and dielectric binary layers to control the emission based on Green's function technique together with the fluctuation-dissipation theorem. The selective emission can be obtained in the infrared and visible range by adjusting the period. An infrared one-dimensional photonic crystal emitter was proposed by Lee et al., ${ }^{57}$ which consisted of a $\mathrm{SiC}$ and photonic crystal structure consisting of dielectric binary layers. The surface wave can be excited in $\mathrm{SiC}$ by the evanescent waves existing in the photonic band gaps by appropriately tuning the lattice constant (or period), thus leading to very narrow emission bands. However, results also showed that these thermal emitters based on 1D PC are relatively sensitive to the polarization of incident light.

Two-dimensional PC can also serve as narrow-band thermal emitters. In fact, 2D PCs can be also referred to as 2D gratings, which are both composed of wavelength-scale periodic arrangement of posts or cavities. ${ }^{34,58}$ As a result, for 2D PCs, design rules based on theoretical mechanisms like the excitation of surface waves using diffraction modes and cavity resonance modes can be applied. On this basis, Pralle et $a l^{58}$ designed a narrow-band $(\delta \lambda / \lambda \leq 0.2)$ thermal emitter which works at mid-infrared frequencies. The developed emitter can be defined by the periodicity of a metal coated silicon-air photonic crystal etched into the emitter surface, and the fabricated PC structure is demonstrated in the inset of left panel of Fig. 2(b). It was found the designed emitters were insensitive to the polarization and incident angle $\left(0-10^{\circ}\right)$, as shown by the reflectance spectra given in the left panel of Fig. 2(b). The experimentally measured thermally emitted power for different PC lattice constants are presented in the right panel of Fig. 2(b). Compared with blackbody radiation, these designed PC emitters are indeed narrowband thanks to the excitation of surface plasmon modes. Similar thermal emitters constructed by combining metallic coating and 2D PhC were proposed in Refs. [59,60]. Meanwhile, Laroche et al. ${ }^{61}$ developed a thermal emitter based on a truncated 2D photonic crystal of infinite germanium (Ge) square rods. The calculated absorption and transmission results by means of the RCWA method revealed that the coherence caused by truncation in structure leads to a directional and quasi-monochromatic emission. Since 2D PCs can be viewed as an array of wavelength-scale multimode resonators, Ghebrebrhan et $a l^{62}$ proposed a $Q$-matching approach combined with the coupled-mode theory to quickly optimize the geometric parameters and subsequently predict the emissivity spectrum of these photonic crystals, which agreed well with FDTD simulations.

Furthermore, a more comprehensive control of photonic band structures can be achieved by thermal emitters based on 3D PCs. As a typical 3D PC, woodpile structure thermal emitters were extensively studied in the works by Fleming et $a l^{63,65,66}$ which can effectively suppress light propagation in all-direction near the emission bands due to a complete (namely, polarization-independent) band gap. A typical 3D PC emitter made of tungsten is presented in Fig. 2(c), ${ }^{63}$ whose experimentally measured thermal radiation power spectra at different temperatures are given in the right panel of Fig. 2(c). High thermal emission peaks occur in the vicinity of the photonic band edge, which are due to the slow light speed and strong percolation through the 3D structure of electromagnetic energy at this wavelength. To overcome the fabrication difficulty of woodpile structure, Han et al..$^{67}$ theoretically proposed that metallic inverse opals, a type of 3D PCs that can be 

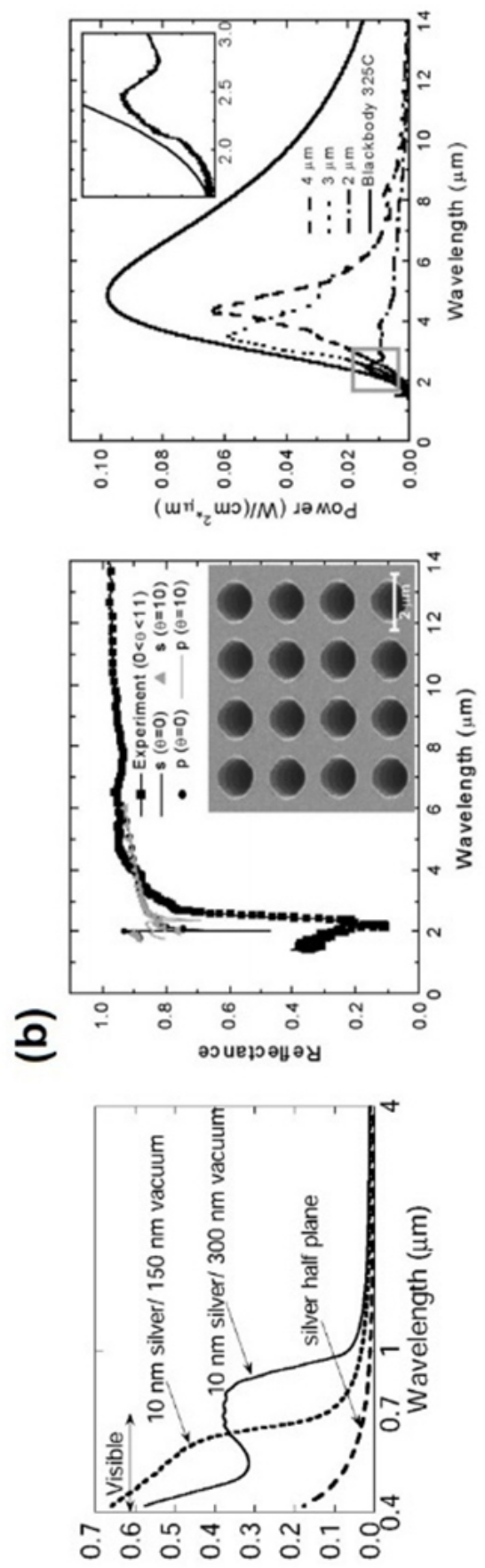

Кำกำรง!̣แ

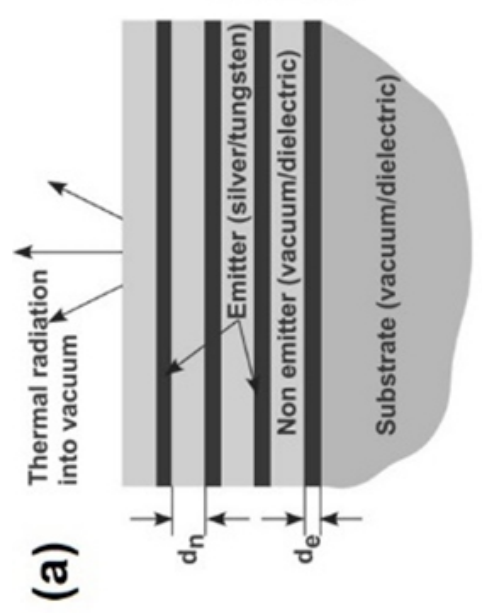

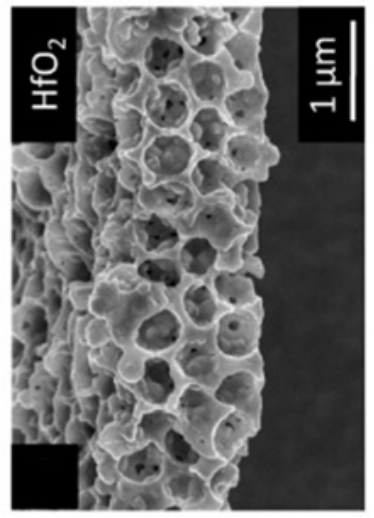

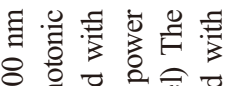

을 可

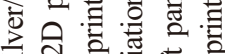

क

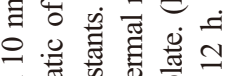

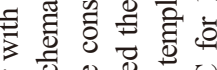

पू

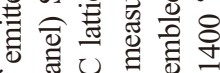

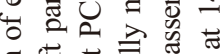

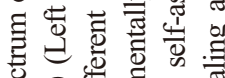

क्षेटी

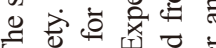

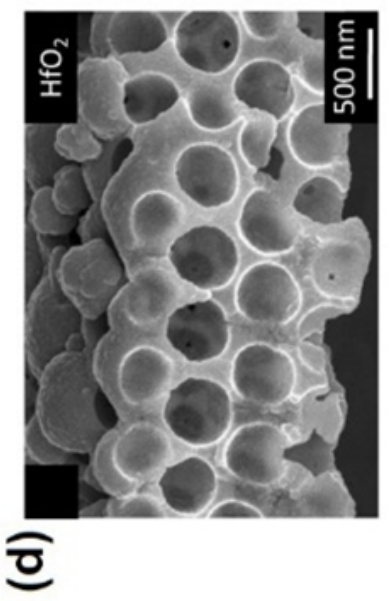

啳

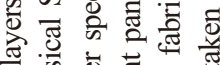

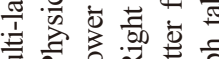

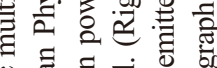

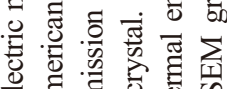

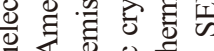

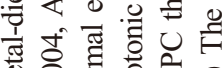

छั 월

字茄

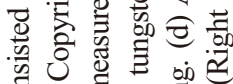

○ं

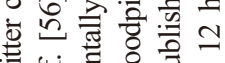

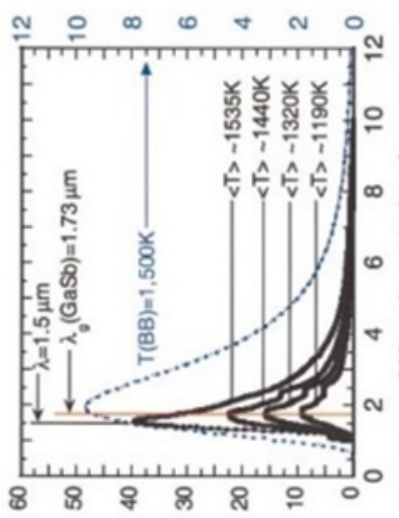

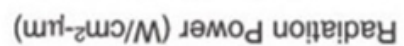

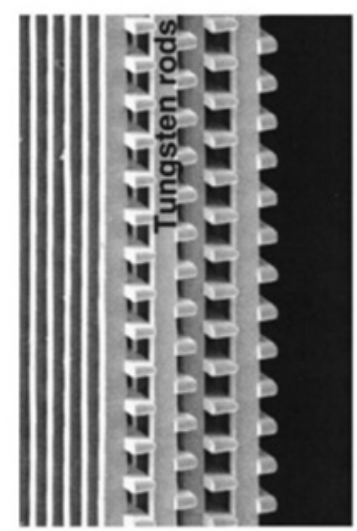

U

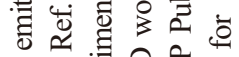

范总会。

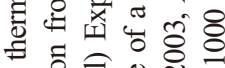

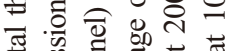

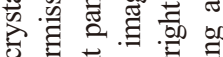

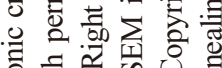

플

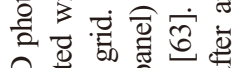

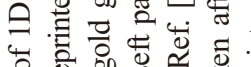

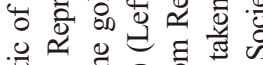

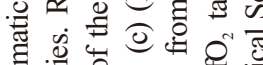

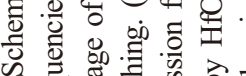

ङ

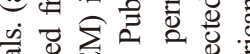

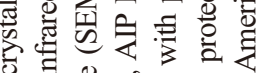

ठ․ㅠ

:

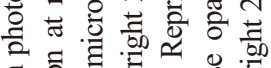

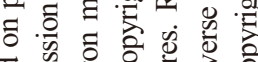

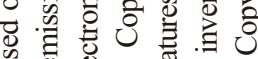

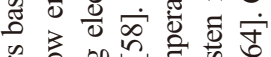

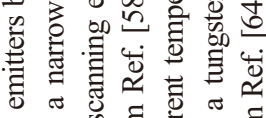

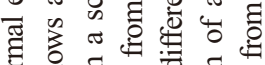

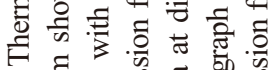

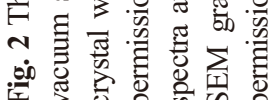


fabricated using the much simpler self-assembling method, may exhibit similar or even better thermal emission performance. Arpin et al. ${ }^{68}$ fabricated this kind of 3D PC thermal made of tungsten using selfassembled templates, which demonstrated a spectral selective thermal emissivity suitable for solar thermophotovoltaic at high operating temperatures. Notably, the fabricated 3D PC by Arpin et al. ${ }^{68}$ exhibited a good thermal stability up to at least $1400{ }^{\circ} \mathrm{C}$, as shown in the left and right panels of Fig. 2(d), in which the microstructures of the tungsten inverse opal PC protected with hafnia are presented after heating to $1000{ }^{\circ} \mathrm{C}$ and $1400{ }^{\circ} \mathrm{C}$ for $12 \mathrm{~h}$, respectively. ${ }^{64}$ These $3 \mathrm{D}$ PC thermal emitters have a great potential in realizing high-efficiency thermophotovoltaics and flexibly tailoring emission profiles.

\section{Emitters based on metamaterials}

Along with the rapid development of electromagnetic metamaterials, the attention of microstructured thermal emitter researchers was very much paid to the use this kind of artificial materials exhibiting exotic properties like negative refraction, ${ }^{2}$ optical cloaking, ${ }^{5}$ spontaneous emission enhancement ${ }^{69}$ and so on, which provide unprecedented possibilities for thermal engineers to tailor thermal radiation. The distinction between the metamaterial and photonic crystal is that the former is usually composed of micro/nanostructures that are substantially smaller than the wavelength of interest. ${ }^{70}$ In most cases, the functionality of metamaterials does not rely on the formation of photonic band gaps (although some locally resonant metamaterials also exhibit photonic band gaps as a result of strong coupling, see Refs. [71, 72], which are out of the scope of the present article.). In terms of the transition from photonic crystal to metamaterials, one can refer to Ref. [73].

In this section, we first introduce the nowadays prevalent metalinsulator-metal (MIM) metamaterials with various micro/nanostructured top metallic layer and discuss the underlying couple mechanism. Then we emphasize two closely related metamaterials, namely, epsilon-nearzero (ENZ) and hyperbolic metamaterials, which exhibit exotic thermal emission behaviors like optical topological transition and directive superPlanckian emission due to the unique properties of effective permittivity. Finally, we briefly review some disordered micro/nanostructures including Mie-resonance based disordered metamaterials and micro/nanoporous textiles.

One seminal experimental work of metamaterial emitters is the one proposed by Liu et al. ${ }^{74}$ In that work, they designed a typical MIM three-layer structure composed of a cross-shaped gold resonator and a gold substrate, with a silicon layer sandwiched between the two gold components. Based on the impedance matching principle (that is, by matching the impedance $Z(\omega)=\sqrt{\varepsilon_{\mathrm{eff}}(\omega) / \mu_{\mathrm{eff}}(\omega)}$ of the metamaterial to free space and thus minimizing the reflectance, where $\varepsilon_{\mathrm{eff}}(\omega)$ and $\mu_{\mathrm{eff}}(\omega)$ are effective permittivity and permeability depending on the electric and magnetic resonances ${ }^{75}$ ), single-band and two-band perfect absorbers were achieved, which had a single resonator and four bipartite resonators in a unit cell, respectively. According to the Kirchhoff's law, single-band and two-band high-performance, wavelength-selective emitters were realized and experimentally verified, whose spectral emissivity is shown in Fig. 3(a). Note in such MIM structures, the coupling mechanism between the metallic resonators and the metallic (a)

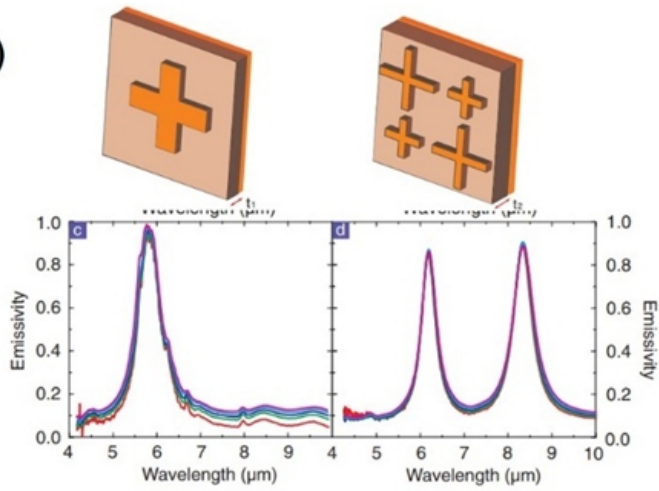

(c)

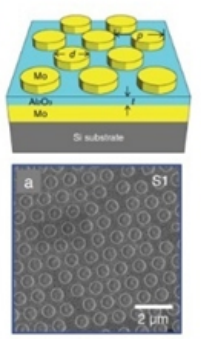

(b)
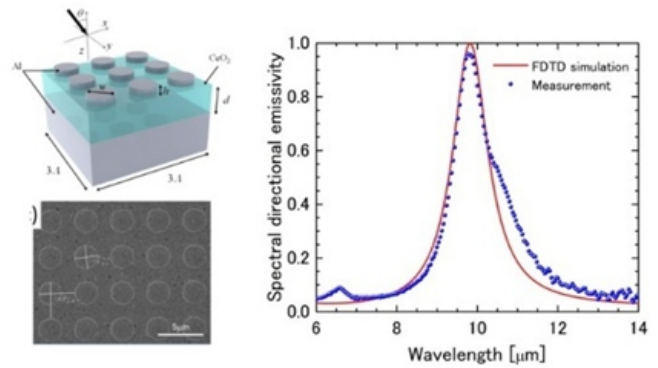

(d)
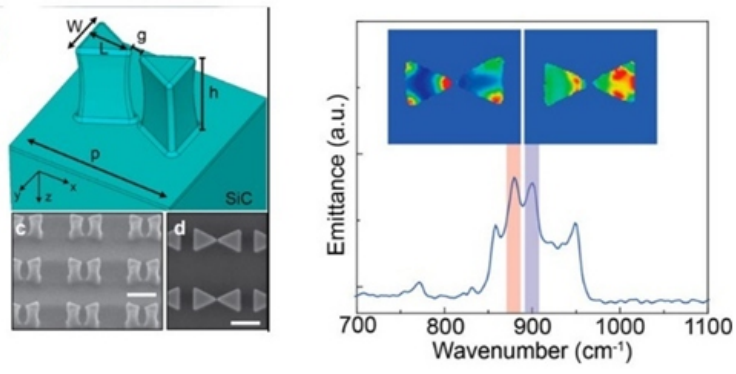

Fig. 3 Typical metamaterial thermal emitters. (a) (Top panel) Schematics of a metamaterial emitter composed of one (left) and four (right) cross-shaped gold resonators and a gold substrate with a sandwiched silicon layer. (Bottom panel) Experimental results for the single-band (left) and two-band (right) perfect thermal emitters. Reprinted with permission from Ref. [74]. Copyright 2011, American Physical Society. (b) A near-perfect emission mid-infrared metamaterial structure. (Left panel) Schematic and SEM graph of the metamaterial emitter. (Right panel) Measured and simulated emissivity spectra. Reprinted with permission from Ref. [77]. Copyright 2017, Optical Society of America. (c) A spectrally selective mid-infrared metamaterial thermal emitter based on molybdenum. (Left panel) Schematic and SEM graph of the metamaterial. (Right panel) Thermal emission intensity spectra at different operating temperatures. Reprinted with permission from Ref. [78]. Copyright 2016, Wiley-VCH Verlag GmbH \& Co. KGaA, Weinheim. (d) A narrowband metamaterial emitter. (Left panel) Schematic and SEM graph of the metamaterial emitter which consists of SiC bowtie resonators with nanoscale gaps. (Right panel) Emittance spectrum with two insets showing the near-field images near the two emission peaks. Reprinted with permission from Ref. [80]. Copyright 2017, American Chemical Society. 
substrate is important to the emission/absorption spectrum, and it depends significantly on the thickness of the intermediate dielectric spacer, which can give rise to a transition between the electromagnetic induced transparency (EIT) and electromagnetic induced absorption (EIA) behaviors. ${ }^{76}$ Matsuno and Sakurai ${ }^{77}$ presented a large-area metamaterial that exhibits near-unity emissivity near $10 \mu \mathrm{m}$. This metamaterial is composed of a $2 \mathrm{D}$ array of $\mathrm{Al}$ nanodisks and an $\mathrm{Al}$ substrate with a $\mathrm{CeO}_{2}$ layer sandwiched between them, as schematically shown in Fig. 3(b). This metamaterial can be fabricated by a metallic patterning process employing photomask and wet etching technologies, which enables the mass-production of a large-area metamaterial in a relatively short time. A SEM graph is also presented in Fig. 3(b) along with the measured and simulated emissivity spectra. Based on an equivalent electric circuit model and the impedance matching condition, it was demonstrated that the narrowband emission is a result of the excitation of magnetic polaritons that arise from antiparallel oscillating currents occur in the top and bottom metallic components. Similarly, Yokoyama et al. $^{78}$ fabricated wavelength-selective thermal emitters based on the MIM metamaterial structure, in which molybdenum and aluminum oxide are used as refractory components to realize both narrow-band infrared thermal emission and excellent thermal stability, as shown in the left panel of Fig. 3(c). The right panel of Fig. 3(c) gives the measured emission intensity spectra at different temperatures, where a good narrowband emission profile is observed. Gong et al. ${ }^{79}$ proposed and investigated a broadband, angle/polarization-insensitive metamaterial thermal emitter with averaged emissivity of $\sim 0.94$ in the mid-infrared atmospheric window of $8-14 \mu \mathrm{m}$. This metamaterial consists of $\mathrm{Cr}$ micro-cuboids on top of tantalum pentoxide $\left(\mathrm{Ta}_{2} \mathrm{O}_{5}\right)$ and silicon dioxide $\left(\mathrm{SiO}_{2}\right)$ thin films. This choice of materials allows this metamaterial to work at relatively high temperatures (over 1000K). Wang et al. ${ }^{80}$ proposed that bowtie antennas made of $\mathrm{SiC}$ with nanoscale gaps can be used as the basic unit for narrowband metamaterial emitters, which utilizes nanoscale coupling effects of adjacent phonon polaritonic excitations in $\mathrm{SiC}$ antennas. The thermal emission frequency can be tuned by the gap distance while the emission linewidth can be retained as narrow as $10 \mathrm{~cm}^{-1}$. The schematic as well as fabricated nanostructure and the emittance spectrum is presented in Fig. 3(d). In particular, at an elevated temperature of $350{ }^{\circ} \mathrm{C}$, four narrowband emission modes were observed with high quality factors around 60 or even higher in this work. A scattering-type scanning nearfield optical microscope (s-SNOM) was implemented to investigate the modal structure.

A notable category of metamaterial emitters is the epsilon-nearzero metamaterial, ${ }^{81}$ whose effective permittivity approaches to zero at a certain wavelength and thus exhibits a strong field enhancement in the metamaterial according boundary condition of continuous normal displacement vectors. ${ }^{82}$ In a seminal work, Molesky et al. ${ }^{83}$ proposed the use of ENZ metamaterials as high-performance emitters for TPV applications. The rigorous concept of ENZ is relaxed into the one with near-zero real part of (effective) permittivity while the imaginary part of permittivity is high. In this condition, the electromagnetic field in the ENZ metamaterial becomes large, resulting in strong absorption if $\operatorname{Im}(\varepsilon)$ is large. They proposed two types of uniaxial ENZ metamaterial thermal emitters, in which the first is the multilayered structure composed of alternating metallic and dielectric layers and the second is the metallic nanowire arrays in dielectric matrix, as shown schematically in the first two panels of Fig. 4(a). The parallel component of the effective permittivity tensor $\varepsilon_{\|}$of multilayered ENZ (a)

(b)
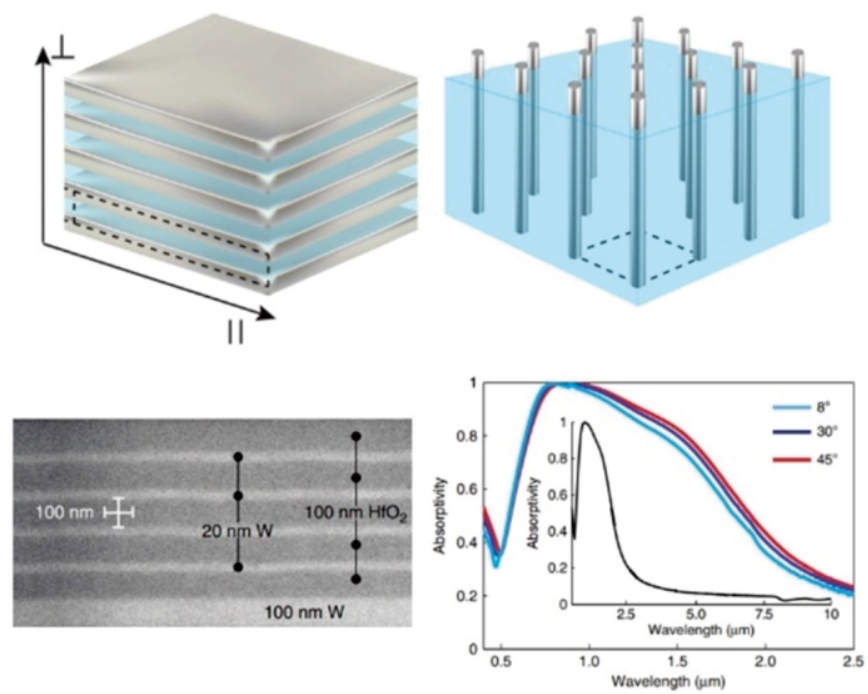
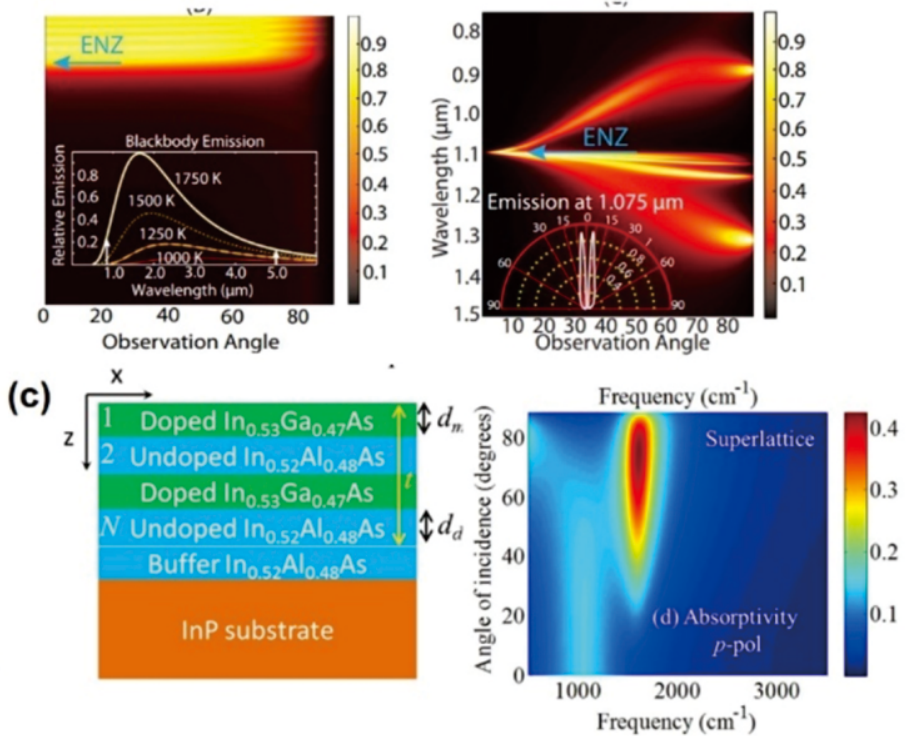

Fig. 4 Epsilon-near-zero and hyperbolic metamaterial emitters. (a) Schematics and emission spectra of two types of uniaxial ENZ metamaterial thermal emitters. (First panel) The multilayered structure composed of alternating metallic and dielectric layers. (Second panel) Metallic nanowire arrays in dielectric matrix. (Third panel) Calculated emission spectra under different observation angles for the multilayered metamaterial with an inset of the relative emission power at different temperatures. (Fourth panel) Calculated emission spectra under different observation angles for the nanowire metamaterial, with an inset of angle-resolved emission profile at $1.075 \mu \mathrm{m}$. Reprinted with permission from Ref. [83]. Copyright 2013, Optical Society of America. (b) (Left panel) The fabricated nanostructure and (Right panel) absorptivity/emissivity of multilayered metamaterials under different incident/emission angles. (Inset) Measured absorptivity of the metamaterial at a $13^{\circ}$ angle of incidence from 0.5 to $10 \mu \mathrm{m}$. Reprinted with permission from Ref. [84] under the terms of the Creative Commons Attribution 4.0 International License. (c) A monochromatic and directional thermal emitter composed of alternating $\mathrm{In}_{0.53} \mathrm{Ga}_{0.47} \mathrm{As}$ and undoped $\mathrm{Al}_{0.48} \mathrm{In}_{0.52} \mathrm{As}$ layers. (Left panel) Schematic of the metamaterial. Right panel: calculated absorptivity/emissivity spectra under different angles of incidence/emission. Reprinted with permission from Ref. [87] under the terms of the Creative Commons Attribution 4.0 International License. 
metamaterial can be near zero, thus leading to an omnidirectional, polarization-insensitive emitter, as shown in the third panel of Fig. 4(a). On the other hand, the nanowire metamaterial exhibited an ENZ behavior for the perpendicular component of the effective permittivity tensor $\varepsilon_{\perp}$, resulting in a highly directional far-field emission profile, presented in the rightmost panel of Fig. 4(a).

Following from this work, Dyachenko et al. ${ }^{84}$ proposed a $\mathrm{W} / \mathrm{HfO}_{2}$ multilayered metamaterial with wavelength-selective thermal emission, which arises from the ENZ behavior associated with the optical topological transition (OTT) of the effective permittivity. The fabricated nanostructure is presented in the left panel of Fig. 4(b). The OTT indicates a specific transition that at a certain wavelength the equifrequency surface changes from an ellipsoid to a hyperboloid, and the critical wavelength is indeed the ENZ point of the parallel effective permittivity $\varepsilon_{1}$, i.e., $\operatorname{Re} \varepsilon_{1}=0$. This metamaterial showed high absorptivity/emissivity below the OTT wavelength, which is also largely independent of the incident/emission angle, as illustrated in Fig. 4(b). Above the OTT wavelength, as thermally excited modes cannot couple to the vacuum modes efficiently, the emissivity is strongly suppressed. Since the OTT wavelength can be tailored by changing the thicknesses of the metallic and dielectric layers as well as composing materials, wavelength-selective absorbers/emitters can be properly designed based on this concept. Many works have been conducted along this line, for example, Refs. $[85,86]$. On the other hand, by utilizing another ENZ condition for uniaxial metamaterials, $\varepsilon_{\perp} \rightarrow 0$, Campione et al. ${ }^{87}$ realized a strong monochromatic and directional thermal emission based on alternating 50 pairs of 10 -nm-thick $\operatorname{In}_{0.53} \mathrm{Ga}_{0.47} \mathrm{As}$ and 8-nm-thick undoped $\mathrm{Al}_{0.48} \mathrm{In}_{0.52}$ As layers, shown in the left panel of Fig. 4(c), where the angle-resolved absorptivity/emissivity of $p$-polarization is presented in the right panel of Fig. 4(c). These simulated results were verified by a following experiment.

Indeed, the performance of above uniaxial ENZ metamaterials is closely associated to their hyperbolic equifrequency surfaces near the ENZ point. More specifically, for type-I hyperbolicity we have $\varepsilon_{\perp}<0$ and $\varepsilon_{1}>0$ and the equifrequency surface is a two-sheeted hyperboloid, and for type-II hyperbolicity, we have $\varepsilon_{1}<0$ and $\varepsilon_{1}<0$ and the equifrequency surface is a one-sheeted hyperboloid. Therefore, the ENZ behavior occurs in the vicinity of the OTT wavelength of hyperbolic metamaterials. The ENZ behavior provides a way to enhance the electromagnetic density while the hyperbolicity determines the directionality. In this circumstance, it is possible to achieve highly directional strong emissivity, and even super-Planckian emission in certain directions can be realized. For instance, Nefedov and Melnikov ${ }^{88}$ theoretically demonstrated the realization of strong directive thermal emission in the far field using asymmetric hyperbolic metamaterials, exceeding that predicted by Planck's limit by over two orders of magnitude in the enhanced direction, where the asymmetry is introduced by tilting the optical axis of the uniaxial hyperbolic metamaterial to enhance the directionality. Note generally, for a macroscopic object, the total far-field emission cannot exceed the (a)
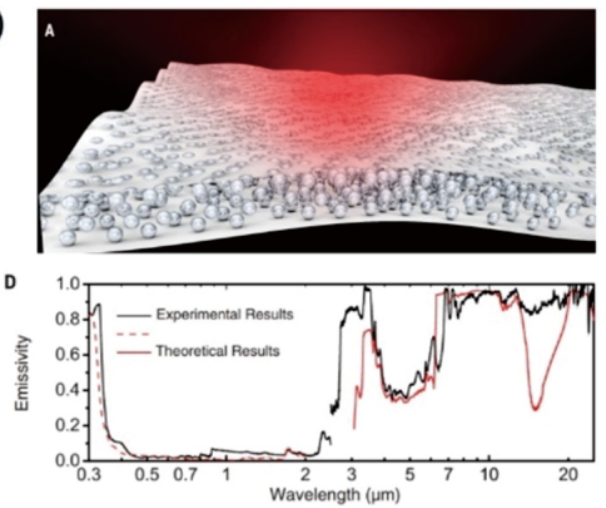

(c)

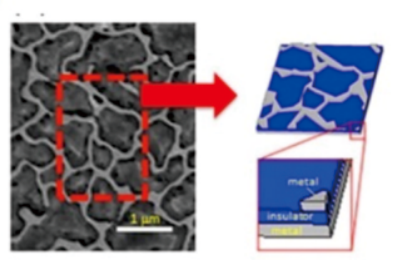

(b)

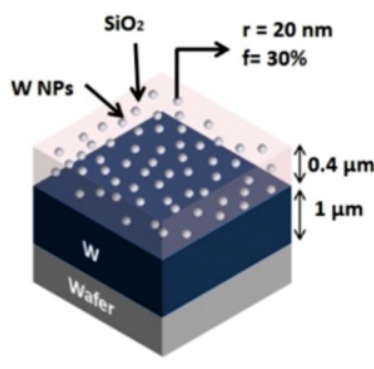

(d)

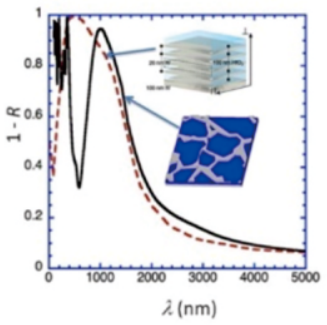

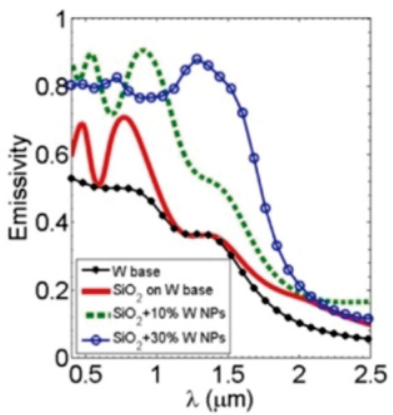
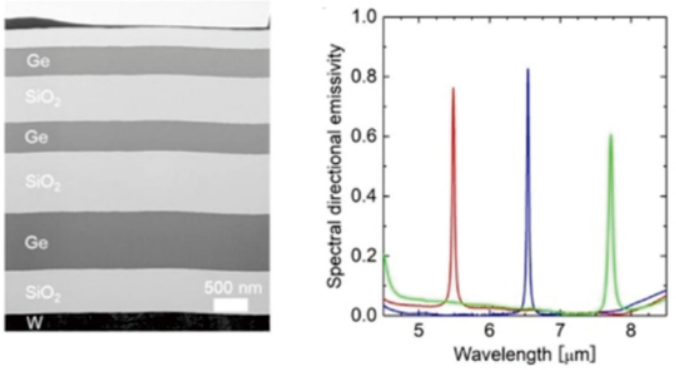

Fig. 5 Disordered/aperiodic metamaterial emitters. (a) (Top panel) A schematic of the polymer-based hybrid metamaterial with randomly distributed SiO ${ }_{2}$ microsphere inclusions. (Bottom panel) Theoretical and experimental emissivity spectra of the disordered metamaterial. Reprinted with permission from Ref. [14]. Copyright 2017, American Association for the Advancement of Science. (b) (Left panel) A proposed design of thermal emitter, which consists of a $\mathrm{SiO}_{2}$ layer doped with $\mathrm{W}$ nanoparticles on the top of a W layer deposited on the substrate. (Right panel) Emissivity spectra of the W base, a $0.4 \mu \mathrm{m}$ thick $\mathrm{SiO}_{2}$ film deposited on the $\mathrm{W}$ base, and $0.4 \mu \mathrm{m}$-thick $\mathrm{SiO}_{2}$ films doped with $20 \mathrm{~nm}$-radius $\mathrm{W}$ nanoparticles of different volume fractions ( $10 \%$ and $30 \%$ ), deposited on the $\mathrm{W}$ base. Reprinted with permission from Ref.91. Copyright 2016, Optical Society of America. (c) A disordered metamaterial thermal emitter based on random metallic networks. (Left panel) Schematic of this metamaterial, in which a $\mathrm{SiO}_{2}$ layer is sandwiched between a continuous W film and a randomly perforated W film. (Right panel) Emissivity spectrum of this metamaterial, compared with that of the ENZ metamaterial in Ref. [84]. Reprinted with permission from Ref. [94]. Copyright 2018, Wiley-VCH Verlag GmbH \& Co. KGaA, Weinheim. (d) Aperiodic ultranarrowband thermal emitter designed from Bayesian optimization. (Left panel) The TEM graph of the fabricated optimal structure operating at $6 \mu \mathrm{m}$ and (Right panel) the experimentally measured emissivity spectra. Reprinted with permission from Ref. [95]. Copyright 2019, American Chemical Society. 
energy flux defined by the Planck's law, ${ }^{89}$ even when the hyperbolic modes are outcoupled by a grating, as recently demonstrated by Sohr et $a l^{90}$

Above metamaterial emitters are all based on periodic micro/nanostructures. Recent years a great deal of attention has been paid to those metamaterials with aperiodic or even random micro/nanostructures, which are comparatively easier to fabricate in a large scale and have a high tolerance to fabrication errors. This type of metamaterials usually relies on the resonances in a single composing unit because the inter-unit coupling effect is difficult to control deterministically in a disordered ensemble. For example, Zhai et al. ${ }^{14}$ proposed a scalable disordered metamaterial based on a polymeric thin film embedded with randomly distributed $\mathrm{SiO}_{2}$ microspheres, which can act as radiative cooling emitters, having an infrared emissivity greater than 0.93 across the atmospheric window. This metamaterial can be manufactured by a high-throughput, economical roll-to-roll method. The schematic of this metamaterial emitter and theoretical and experimental emissivity spectra are shown in Fig. 5(a). For this metamaterial, the high performance in the mid-infrared emissivity is attributed to the localized phonon polaritons supported by the $\mathrm{SiO}_{2}$ microspheres. Ghanekar et al. ${ }^{91}$ proposed a Mie-resonance based metamaterial thermal emitter for thermophotovoltaic applications, which consists of a thin film of $\mathrm{SiO}_{2}$ embedded with $\mathrm{W}$ nanoparticles on the top of tungsten layer, as shown in the left panel of Fig. 5(b). A broadband high emissivity (presented in the right panel of Fig. 5(b)) was achieved in the wavelength range of $0.4 \mu \mathrm{m}$ to $2 \mu \mathrm{m}$, which is suitable for $\mathrm{GaSb}$ and InGaAs based photovoltaic cells. This enhancement of emissivity is due to the Mie resonances in the $\mathrm{W}$ nanoparticles, which can be captured by a Maxwell-Garnett-Mie effective medium model. In this model, the effective permittivity is expressed as

$$
\frac{\varepsilon_{\mathrm{eff}}-1}{\varepsilon_{\mathrm{eff}}+2}=\frac{2 \pi i}{k^{3}} \rho a_{1}
$$

in which $a_{1}$ is the first-order Mie coefficient denoting the electric dipole excitation, $k$ is the wavenumber in the background medium and $\rho$ is the number density of nanoparticles for monodispersed media. A similar formula can be obtained for effective permeability if the magnetic dipole is excited. Note these formulas are derived on the basis of the local field approximation for randomly distributed nanoparticles. ${ }^{92}$ In addition, another notable work using Mie resonances to enhance thermal emission is the one conducted by Asano et al., ${ }^{93}$ which combined the interband transitions and the Mie resonances in intrinsic Si nanorods to realize a high thermal emissivity in the near-infrared-tovisible range. Although the experimentally fabricated structure was composed of ordered arrays of $\mathrm{Si}$ nanorods, it is expected that a disordered array can also exhibit a comparably high emissivity because the performance mainly depends on the optical response of individual nanorods rather than their spatial arrangements.

Micro/nanoporous materials can also be used as disordered metamaterial emitters. For instance, Kong et al..$^{94}$ proposed a random metamaterial thermal emitter based on three-layer structures, which is composed of an insulator $\left(\mathrm{SiO}_{2}\right)$ layer sandwiched between a continuous metallic (W) film and a randomly perforated metallic film, schematically shown in the left panel of Fig. 5(c). In the right panel of Fig. 5(c), it is demonstrated that this metamaterial is able to strongly absorb/emit electromagnetic radiation in a narrow band (centered around $1 \mu \mathrm{m}$ ) due to the near perfect impedance matching to the vacuum impedance. Cui and coworkers ${ }^{96,97}$ demonstrated nanoporous polyethylene (nanoPE) microfibers can be tailored to achieve a low thermal emission and high transmission spectrum in the midinfrared atmospheric transparency window, leading to radiative cooling textiles for human skin. Other textile materials like porous poly(vinylidene fluoride-cohexafluoropropene) $\left[\mathrm{P}(\mathrm{VdF}-\mathrm{HFP})_{\mathrm{HP}}\right]$ coatings $^{98}$ were also investigated. In addition, it is also interesting that vanadium dioxide $\left(\mathrm{VO}_{2}\right)$ can be regarded as a natural disordered metamaterial ${ }^{99}$ when it is at an intermediate state of its metal-insulator transition determined by temperature, which comprises nanoscale islands of metal and dielectric phases. This property allows people to control the thermal emission spectra by temperature. Another recently notable work is the aperiodic multilayered metamaterial emitter proposed through the Bayesian optimization method, which is a design algorithm based on machine learning. ${ }^{95}$ In that work, in order to achieve an ultranarrow-band wavelength-selective thermal emitter, the optimal configuration of multilayers was efficiently identified from over 8 billion candidates consisting of multiple components $\left(\mathrm{Si}, \mathrm{Ge}\right.$, and $\left.\mathrm{SiO}_{2}\right)$ for a given target infrared wavelength. The resulting optimized structure is an aperiodic multilayered metamaterial exhibiting high and narrow-band emissivity with a quality factor of 273. Fig. 5(d) shows the TEM graph of the fabricated optimal structure operating at $6 \mu \mathrm{m}$ and the experimentally measured emissivity spectra.

\section{Emitters based on metasurfaces}

Metasurfaces are usually regarded as a new branch of metamaterials in two dimensions, which are generally composed of planar and subwavelength meta-atoms (which can be resonant or not) in a periodic or aperiodic arrangement. ${ }^{100-103}$ The thickness of a metasurface is usually ultrathin with respect to the wavelength of interest (that is, optically thin). Specifically, the electromagnetic response of the whole configuration can be artificially tailored by engineering an individual resonant meta-atom, including the shape, size and composite material, and also the spatial and orientational arrangements of these metaatoms. ${ }^{10-103}$ In the field of nanophotonics, we have witnessed the rapid development of metasurfaces in the past several years, which provide unprecedented opportunities of manipulating the amplitude, ${ }^{104}$ phase, ${ }^{105}$ polarization $^{106}$ of electromagnetic waves and foster exciting applications in imaging, ${ }^{107}$ sensing, ${ }^{108}$ information processing ${ }^{109}$ and energy harvesting and conversion. ${ }^{110}$

Since we note in many works, MIM structures are also called metasurfaces, e.g., Refs. [16, 111-114], which are already discussed in previous sections and very common in designing thermal emitters, ${ }^{16,11-114}$ herein we do not review these works. In fact, the distinction between the metasurface and 2D grating and photonic crystal is that the former is composed of optically thin planar subwavelength micro/nanostructures. In this sense, some thin MIM structures composed of wavelength-scale units cannot be regarded as standard metasurfaces although they might also be optically thin, e.g., Ref. [112]. The most confusing type is those MIM structures with subwavelength-scale elements, e.g., Ref. [66]. However, for these structures, the coupling between top metallic structures and bottom metallic layers is critical for the performance. They cannot be regarded as metasurfaces either, because the working principle is fundamentally different from standard metasurfaces. In other words, these structures can only be dubbed "planar metamaterials". ${ }^{115}$ In addition, they are also different from the so-called few-layer metasurfaces in which each individual metasurface layer can work independently. ${ }^{116-118}$ Therefore, in this review, according to the scale of composing elements with respect to the wavelength, 2D MIM structures can be classified as gratings or metamaterials. In Table I presented in the Appendix, we also give a simple classification of different micro/nanostructures based on the characteristic length scales of three dimensions. 
Consequently, in the section, we mainly review two types of metasurface-based thermal emitters, including all-dielectric and phasegradient metasurfaces. They are composed of planar subwavelengthscale elements, and have been extensively studied in the nanophotonics community but received less attention in the thermal radiation community. They indeed show promising prospects for compatible integration in micro/nano devices for thermal radiation control.

Dielectric materials ${ }^{102}$ possessing strong Mie resonances when the characteristic sizes of structures are compared with the incidence wavelength represent an alternative route to control thermal emission/absorption. Although the intrinsic loss is low for dielectric materials, strong microstructure-induced resonances can be designed to tailor thermal emission. For example, by changing the height or diameter of nanopillars and nanocones, Wang et al. ${ }^{119}$ experimentally demonstrated Si-based metasurfaces to achieve broadband absorption/emission covering the whole visible range $(300 \sim 1000 \mathrm{~nm})$. The intrinsic mechanism was attributed to coupling between Mie resonances and Wood-Rayleigh anomaly effects. Similarly, Länk et al. ${ }^{120}$ designed polarization-independent silicon absorber/emitter via tuning the interference between electric and magnetic dipoles of the meta-atoms. In particular, they employed the hole-mask colloidal lithography technique $^{121}$ to fabricate homogeneous metasurfaces with short-range translational order as shown in the left panel of Fig. 6(a), and the right panel of Fig. 6(a) shows the reflectance spectra, which are almost polarization-independent. By exploiting the first Kerker condition (namely, the electric and magnetic dipole are of the same amplitude and interference constructively) combined with lattice resonances, Yang et $a l .{ }^{122}$ proposed an a-Si nanoantenna-based metasurface narrowband high-efficiency absorber/emitter working at near-infrared region as illustrated in Fig. 6(b). In this structure, the spectral overlap between electric and magnetic dipoles (i.e., the first Kerker effect) leads to the high-quality-factor absorption/emission peak.

A unique feature of metasurfaces, fundamentally different from previously discussed metamaterials, is that the meta-atoms can be arranged in a way to induce a phase gradient and geometric phase, which can lead to the generalized laws of refraction and reflection and spin-orbit coupling for electromagnetics, thus achieving more exotic manipulation of light transport than previous micro/nanostructures. ${ }^{125}$ For example, Chalabi et al. ${ }^{123}$ demonstrated a structured SiC surface, in which a phase gradient $\partial \varphi / \partial x$ was inscribed by judiciously designing the distribution of nanostructures, as shown in the left panel of Fig. 6(c). This artificial phase gradient can mimic that of a lens and therefore make the emitted thermal radiation focused. It can be also understood as an extra momentum that adds to the emitted waves. ${ }^{115}$ The performance of this thermal radiation lens is given in the right panel of Fig. 6(c). Based on an elaborate local rotation of the anisotropy axis of $\mathrm{SiC}$ nanorod arrays (left panel of Fig. 6(d)), Dahan et al. ${ }^{124}$ induced a geometric phase (or called Pancharatnam-Berry phase) for the transport of the emitted thermal radiation, resulting in a splitting of dispersion relations for left- and right- circularly polarized radiation. As a result, this metasurface can emit different circularly polarized thermal radiation at different wavelengths, as indicated in the right panel of Fig. 6(d). We envision that this type of metasurfaces using phase gradient and geometric phase can be elaborated to achieve much more versatile thermal radiation control, while only a few works have been conducted.

\section{Reconfigurable thermal emitters}

For most of aforementioned thermal emitters, their emission peaks are usually fixed at certain wavelengths and cannot be actively tuned after fabrication. In order to dynamically manipulate thermal emission and realize multiple functions in a single micro/nanostructure, a variety of (a)

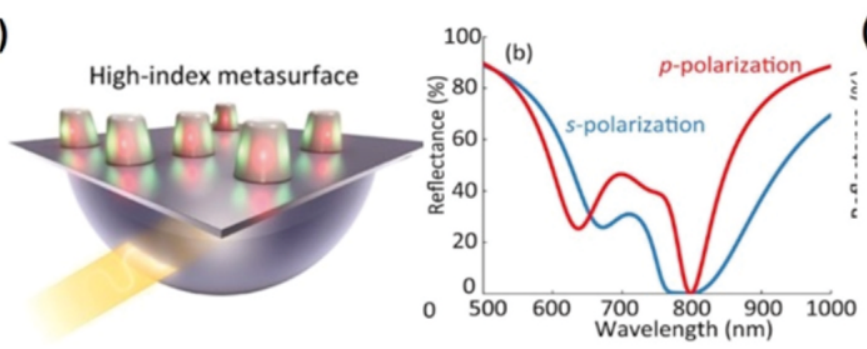

(b)

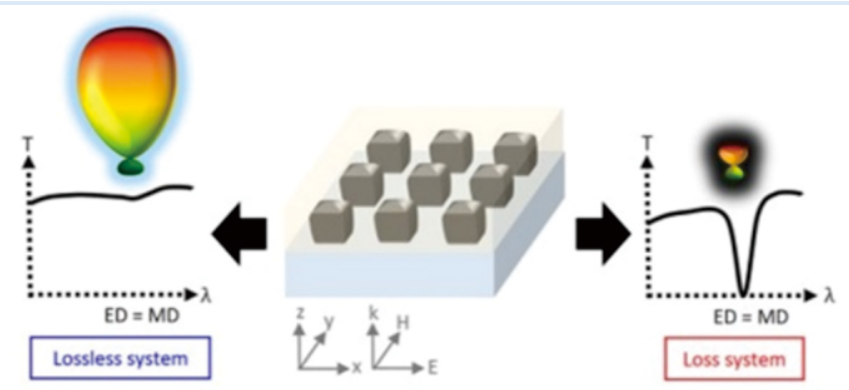

(c)

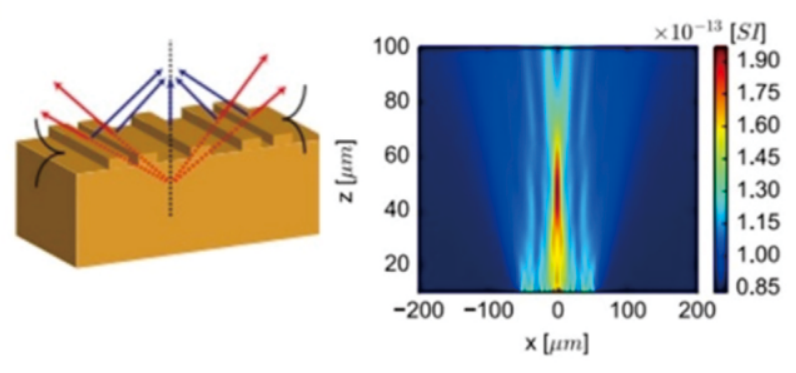

(d)
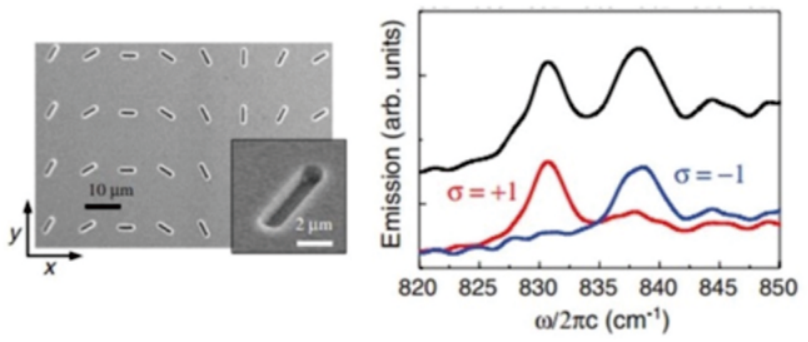

Fig. 6 Metasurface-based thermal emitters. (a) Schematic (Left panel) and reflectance spectra for different polarizations (Right panel) of a dielectric metasurface perfect absorber/emitter composed of Si nanopillars. The metasurface operates in a total internal reflection configuration. Reprinted with permission from Ref. [120]. Copyright 2017, American Chemical Society. (b) Schematic of an a-Si metasurface narrowband absorber/emitter based on the first Kerker condition and lattice resonances, in which the rightmost panel indicates that the overlap of electric dipole (ED) and magnetic dipole (MD) in a lossy system can induce narrowband absorption/emission. Reprinted with permission from Ref. [122]. Copyright 2018, American Chemical Society. (c) (Left panel) Schematic of SiC metasurface to focus thermal radiation. (Right panel) Thermal emission distribution at different height above the surface at the frequency $\omega=1.66 \times 10^{14} \mathrm{rad} / \mathrm{s}$. Reprinted from Ref. [123]. Copyright 2016, American Physical Society. (d) (Left panel) SEM image of the rotated antenna array. (Right panel) Thermal emission spectra for differently circularly polarized radiation, where $\sigma= \pm 1$ stands for right and left polarization, respectively. Reprinted from Ref. [124]. Copyright 2010, American Physical Society. 
methods have been proposed such as mechanical stretching, ${ }^{126}$ electric modulation, ${ }^{127}$ thermal control ${ }^{128}$ and so on. For a recent review on reconfigurable metamaterials/metasurfaces, one can refer to Ref. [129]. Here we only focus on the dynamic modulation on thermal radiation.

Thermal control is one of the most commonly used methods to tune the thermal emissivity of designed emitters, since there are some available materials whose optical properties strongly depend on the temperature, like $\mathrm{VO}_{2}$ and $\mathrm{Ge}_{2} \mathrm{Sb}_{2} \mathrm{Te}_{5}$ (GST). For example, Sun et al. ${ }^{130}$ designed a $\mathrm{VO}_{2}$-based thermal emitter, as shown in Fig. 7(a), exhibiting a dual-band high emissivity in the two atmospheric windows (3-5 $\mu \mathrm{m}$ and 8-14 $\mu \mathrm{m}$ ) at room temperature, while at high temperature, the highemissivity band can be shifted to $5-8 \mu \mathrm{m}$. This reconfigurable emitter can be flexibly utilized to achieve active radiative cooling. Qu et al. ${ }^{128}$ proposed $\mathrm{Au} / \mathrm{GST} / \mathrm{Au}$ emitters (see Fig. 7(b)), whose maximum emissivity can be higher than 0.7 in experiments. They found when the GST is altered from amorphous phase to crystalline phase via controlled temperature, the coupling between high-order magnetic mode and antireflection mode takes place, thus leading to a spectral shift of emission peak from 6.51 to $9.33 \mu \mathrm{m}$. Similarly, another tunable thermal emitter working in the range of $2 \sim 4 \mu \mathrm{m}$ based on $\mathrm{Au} / \mathrm{SiO}_{2} / \mathrm{GST} / \mathrm{Au}$ was also demonstrated in Ref. [124], in which it was shown that the emissivity can be optimized via the period of upper Au arrays, as presented in Fig. 7(b). Moreover, by taking advantage of strain-induced buckling based on an elastomeric substrate polydimethylsiloxane (PDMS) (see Fig. 7(c)), Moridani et al. ${ }^{119}$ has realized tunable infrared radiation in three wavelengths attributed to controlled periodicity of the plasmonic metasurfaces.

However, the modulation speed of aforementioned methods controlled by temperature or mechanical stretch is relatively low. Electric modulation based on 2D materials, ${ }^{136}$ varactor/PIN diodes, ${ }^{132}$ doped semiconductors, ${ }^{137-139}$ liquid crystals ${ }^{140}$ is a promising way to improve the modulation speed. For example, Vasant et al. ${ }^{132}$ designed an electrically controlled $\mathrm{THz}$ thermal emitter combining with thin GaAs quantum well (QW), as shown in Fig. 7(d). The electron density in QW (layer-b in Fig. 7(d)) and thus its refractive index can be tuned by applying an external voltage between metallic grating (layer-a in Fig.

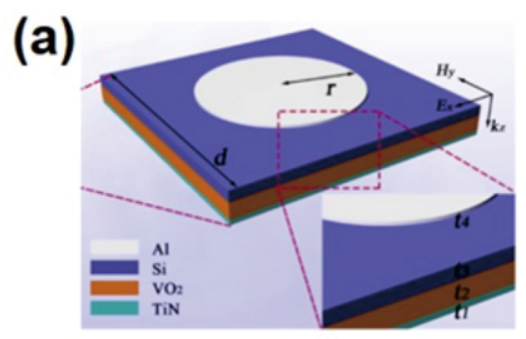

(d)

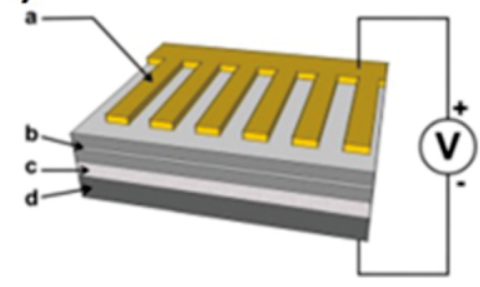

(e)

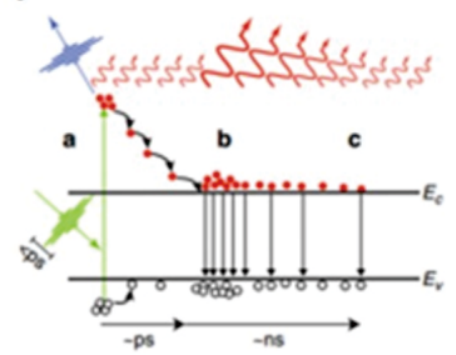

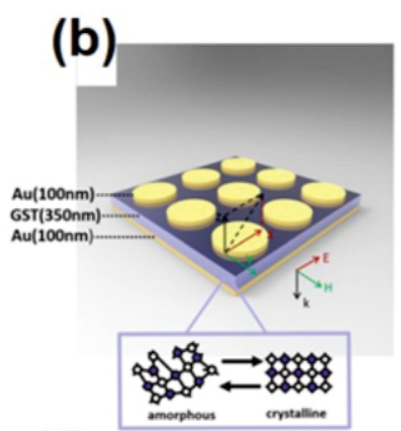

(c)
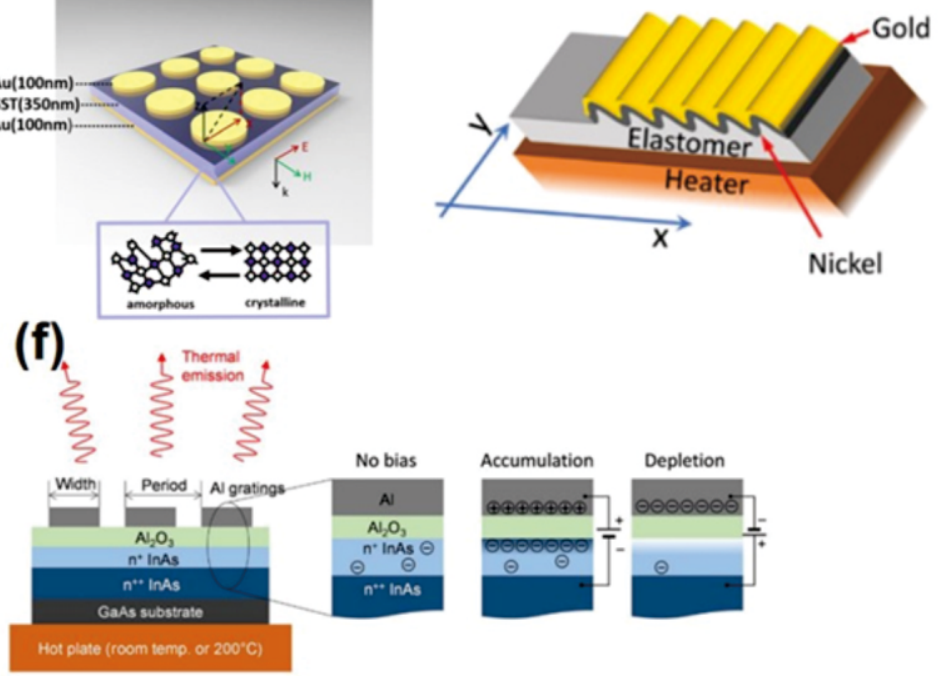

(g)

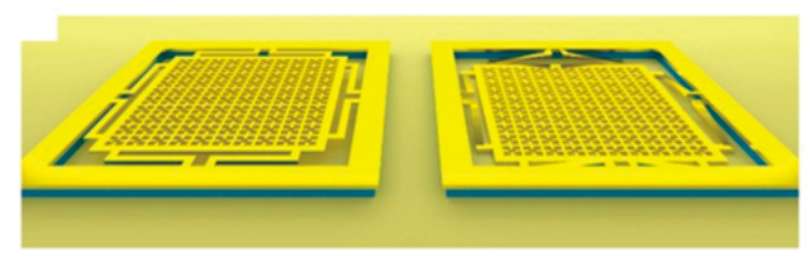

Fig. 7 Reconfigurable thermal emitters. (a) Illustration of a unit cell of $\mathrm{VO}_{2}$-based thermal emitter. Reprinted with permission from Ref.130. Copyright 2019, Optical Society of America. (b) Schematic of the Au/GST/Au emitter. Reprinted with permission from Ref. [128]. Copyright 2017, Wiley-VCH Verlag GmbH \& Co. KGaA, Weinheim. (c) Schematic of the radiometric experiment setup based on elastomeric substrate. Reprinted with permission from Ref. [126]. Copyright 2017, Wiley-VCH Verlag GmbH \& Co. KGaA, Weinheim. (d) Schematic of a THz thermal emitter controlled by a gate voltage, in which $a$ is gold grating, $b$ is a GaAs quantum well, $c$ and $d$ are $\mathrm{Al}_{0.5} \mathrm{Ga}_{0.5} \mathrm{As}$ spacer and n-doped GaAs substrate respectively. A gate voltage is implemented between the grating and the substrate. Reprinted with permission from Ref. [132]. Copyright 2013, AIP Publishing. (e) Schematic of the temporal evolution of thermal emission in photoexcited intrinsic semiconductors. Reprinted with permission from Ref. [133] under the terms of the Creative Commons Attribution 4.0 International License. (f) Configuration of an electrically tunable III-V-based emitter, along with the emissivity spectra under different external conditions. Reprinted with permission from Ref. [134] under the terms of the Creative Commons Attribution 4.0 International License. (g) Schematic of the MEMS metamaterial emitter, including “on” (left panel) and "off” (right panel). Reprinted with permission from Ref. [135]. Copyright 2017, Optical Society of America. 
7(d)) and the substrate (layer-d in Fig. 7(d)), which directly influence the thermally excited $\mathrm{SPhP}$ modes confined in the single $\mathrm{QW}$ and then modulate the emission peak. By combining the multiple QW with photonic crystal slabs, Inoue et al..$^{141}$ presented a realization of dynamic thermal emission control with a high modulation rate. In Fig. 7(e), Luo et $a l^{127}$ proposed to modulate the thermal emission of semiconductors like $\mathrm{Si}$ in time domain. By controlling the carriers in materials, it is promising to design ultrafast thermal emitters under nanosecond modulation, as well as controlled spectral and spatial features. Similarly, Fig. 7(f) demonstrates an electrically tunable thermal emission, proposed by Park et al. ${ }^{134}$ by adding active InAs layers into $\mathrm{Al} / \mathrm{Al}_{2} \mathrm{O}_{3} / \mathrm{Al}$ metasurfaces $(\mathrm{dt} \approx 630 \mathrm{~nm}$ ). The emission peak (around $7.3 \mu \mathrm{m}$ ) can be shifted to short or long wavelength via varying the charge density in low-doped $(\mathrm{n}+)$ InAs layer. In addition, Liu and Padilla ${ }^{135}$ proposed a new route to achieve reconfigurable thermal emission by integrating metamaterials with micro/nanoelectromechanical systems as illustrated in Fig. 7(g). The modulation speed can be as higher as $110 \mathrm{kHz}$, paving an alternate way to realize real-time control of thermal radiation.

\section{Applications}

7.1 Directive, polarized, and/or narrowband thermal emitters The most direct application of micro/nanostructured thermal emitters is to invent novel thermal radiation sources that are totally different from conventional thermal radiators whose emission profile is usually omnidirectional, unpolarized and broadband. A variety of directive, polarized or narrowband thermal emitters have been designed and manufactured in the recent years based on different micro/nanostructures and materials.

One of the earliest works on directive thermal emitters is the one proposed by Greffet et al., ${ }^{32}$ as discussed in Section 2, by using the diffraction of surface phonon polaritons in $\mathrm{SiC}$ gratings. Inampudi and Mosallaei $^{142}$ theoretically proposed a method to mitigate the angular dispersion (change of emission angle with frequency) by inserting bundled graphene sheets into the $\mathrm{SiC}$ substrate and $\mathrm{SiC}$ gratings, as schematically shown in Fig. 8(a). This method achieved a substantially lower angular dispersion of $16^{\circ}$ in a relatively broad wavelength range of $11-12 \mu \mathrm{m}$ than that of a single $\mathrm{SiC}$ grating (about $30^{\circ}$ ), as demonstrated by the lower panel of Fig. 8(a). Moreover, by using a dielectric grating on the $\mathrm{SiC}$ substrate a significantly low angular dispersion around $4^{\circ}$ can be obtained, although in this circumstance the emissivity is reduced.

For narrowband thermal emitters, there are also a great deal of works, some of which are already discussed in previous sections, for example, Refs. [74, 80, 95]. A general method to achieve extremely narrowband thermal emission is to exploit some high-quality-factor electromagnetic resonances. The $\mathrm{SPhPs}$ in $\mathrm{SiC}$, whose quality factor is (a)

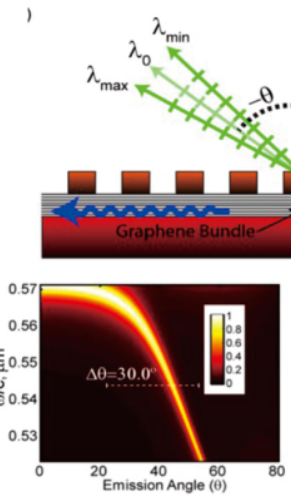

(c)

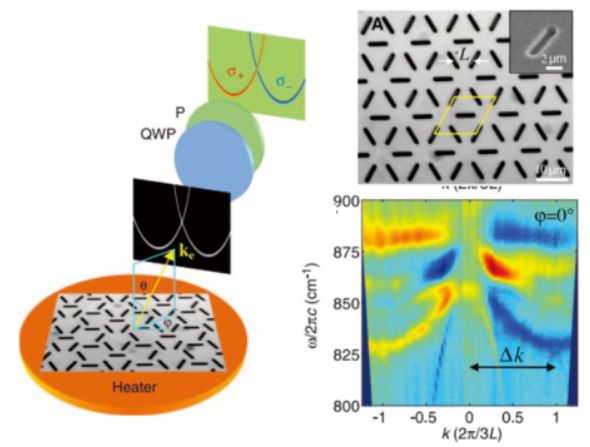

(b)

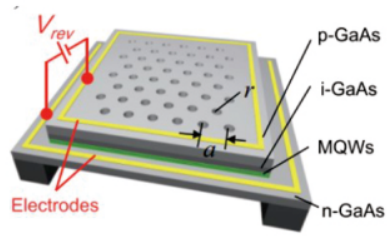

Emitter C: $a=4.5 \mu \mathrm{m}, r=0.12 a$

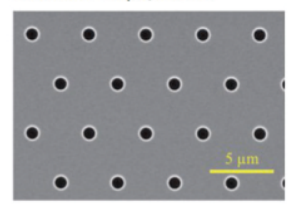

(d)

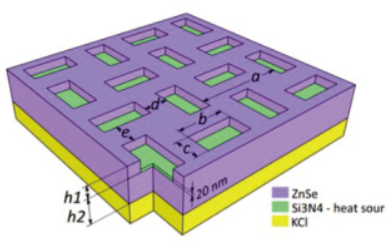

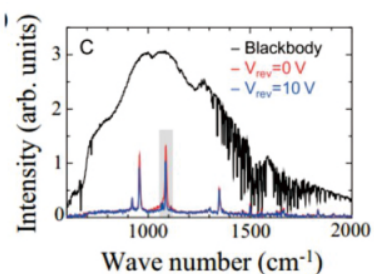

Wave number $\left(\mathrm{cm}^{-1}\right)$
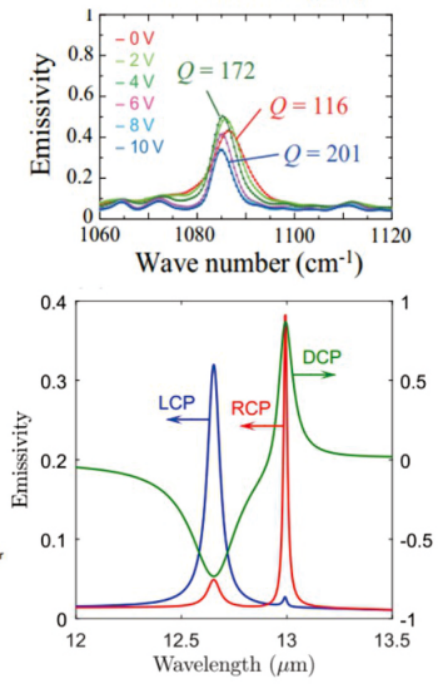

Fig. 8 Directive, narrowband and polarized thermal emitters. (a) (Top panel) A directional thermal emitter composed of a SiC grating and substrate with bundled graphene sandwiched between them. (Bottom panel) The angle-resolved emissivity spectra before and after inserting the bundled graphene. Reprint with permission from Ref. [142]. Copyright 2017, American Physical Society. (b) Ultranarrowband thermal emitters. (Left panel) Schematic and SEM graph of the thermal emitter based on a 2D MQW photonic crystal. (Right panel) Experimentally measured emission intensity and emissivity spectra of the emitter at different bias voltages that tune the ISB absorption, with quality factors given. Reprinted from Ref. [148]. Copyright 2015, American Physical Society. (c) A metasurface thermal emitter that radiates circularly polarized light. (Left panel) The experimental setup to resolve the polarization states of the thermally emitted radiation, where QWP is a quarter-wave plate, P is a linear polarizer. (Right panel) A SEM graph of the metasurface and the measured dispersion relations for the two photon spins. Reprinted with permission from Ref. [152]. Copyright 2013, American Association for the Advancement of Science. (d) A metamaterial that thermally emits circularly polarized radiation in a narrowband. (Left panel) Schematic of the metamaterial with geometric parameters $a=10 \mu \mathrm{m}, b=4 \mu \mathrm{m}, c=2 \mu \mathrm{m}$. (Right panel) Thermal emissivity spectra for LCP and RCP radiation, along with the DCP for right polarized radiation. Reprinted with permission from Ref. [153]. Copyright 2018, American Physical Society. 
around 50-100, are good candidates, while conventional SPPs in metallic nanostructures can only be used for the circumstances that are not demanding in the emission linewidth due to their lower quality factor usually around $10 .^{74}$ However, some elaborately designed plasmonic states with high quality factor, like the Tamm plasmons (TPs), can achieve ultranarrowband thermal emission. For example, a typical TP structure composed of a metal and distributed Bragg reflector (DBR) was experimentally shown to exhibit a narrow thermal emission peak with a quality factor of 36.5. ${ }^{143}$ Moreover, recently Zhu et al ${ }^{144}$ proposed an ultranarrow band thermal emitter based on a bilayer cavityenhanced TP structure, which consists of a high/low refractive index bilayer sandwiched between a metal and DBR. The inserted high/low refractive index bilayer cavity can simultaneously enhance the quality factor and emissivity compared to the conventional DBR-metal TP structure. Theoretical quality factor for this modified structure can achieve $\sim 453$. The experimentally measured quality factor was shown to be $\sim 172$, with a peak emissivity $\sim 0.7$. Another good choice is the ultranarrow intersubband transitions (ISB-Ts) in doped semiconductor QWs whose typical FWHM is around 50 to $100 \mathrm{~cm}^{-1}$. By appropriately modifying the quantum well width, barrier height and materials, the ISB-T wavelength can be tuned from the $\mathrm{THz}$ to near-infrared region, and the absorption coefficient of the ISB-T can be controlled by the doping density. In this circumstance, narrowband thermal emission can be reached by heating the quantum wells. It was demonstrated that the thermal emission spectra of a MQW wafer for both TE and TM waves can reach a linewidth as narrow as $11 \mathrm{meV} .^{145}$ Moreover, by coupling the MQW to photonic crystals and utilizing the band edge resonant effect to achieve $Q$-matching, the linewidth can be further reduced, and the emissivity can be significantly enhanced. Using this method, De Zoysa et $a l .{ }^{145}$ realized an ultranarrowband thermal emitter with an emission peak linewidth as narrow as $4.5 \mathrm{meV}$. Later, improved designs of this type of composite nanostructures were proposed and an emission peak near $1100 \mathrm{~cm}^{-1}$ with an impressively high quality factor over 150 can be obtained. ${ }^{146-148}$ A typical realization of this type of narrowband emitters based on MQW 2D photonic crystals is presented in Fig. 8(b) with a schematic and SEM graph of the fabricated structure, and the experimentally measured emission intensity and emissivity spectra indeed demonstrated high-quality factor peaks. ${ }^{148}$ Moreover, by utilizing the van Hove singularities in the DOS periodic metallic structures, Biener et al. ${ }^{149}$ realized an ultranarrowband thermal emitter based on a metallic grating, which can, in theory, achieve high-quality-factor $(Q=$ 2320) thermal emission near the narrow plasmonic band gap (around $11.6 \mu \mathrm{m}$ ), and a following experiment measured a $Q=460$ emission peak. However, the emissivity is relatively low (around 0.17). Similarly, by critically coupling a flat tungsten surface with the cavity (guided) resonances of a dielectric photonic crystal slab, Guo and Fan ${ }^{150}$ numerically proposed a narrowband thermal emission with a near-unity emissivity peak in the near-infrared range. Ali et $a l^{151}$ recently numerically proposed a kind of all-dielectric midinfrared emitters with a maximal $Q$-factor reaching 650, which is composed of an array of elliptical $\mathrm{Si}$ resonators and $\mathrm{SiO}_{2}$ layer sandwiched between the resonator arrays and a $\mathrm{Cu}$ back reflector. These designs are promising while further experimental verification and demonstration are still needed.

For thermal emitters that can radiate polarized electromagnetic waves, there are also a variety of different schemes. Under the Kirchhoff's law, polarization-dependent emission is equivalent to polarization-dependent absorption. For linearly polarized radiation, Ikeda et $a l^{154}$ experimentally demonstrated a linearly polarized midinfrared $(2.5-5.5 \mu \mathrm{m})$ thermal emitter based on 1D subwavelength gratings of narrow and deep (100-nm-wide, 1000-nm-deep trenches) rectangular cavities engraved on a Au surface. For circularly polarized emitters, Wadsworth et $a l^{155}$ proposed a simple device which consists of a circular polarizer (a meanderline quarter-wave plate) and an emitter, with a transparent thermal insulating layer sandwiched between them. Although this design is straightforward, its energy conversion efficiency is low, and the experimentally measured degree of circular polarization (DOCP) is around $28 \%$ due to the unavoidable thermal emission from the polarizer. A similar device composed of a linearly polarized emitter and a circular polarizer was also proposed by Song et al. ${ }^{156}$ However, it is more desirable to realize an intrinsic circularly polarized thermal emitter to avoid above bulky layer-by-layer structure. Generally, by breaking the mirror symmetry of the system, circularly polarized radiation can be generated. On this basis, two approaches are usually used to achieve this goal. The first is to apply an external magnetic field to induce the magneto-optic Kerr effect, for example, e.g., Ref. [157]. However, it is usually not convenient to apply a large magnetic field, and another natural idea is to resort to some chiral micro/nanostructures. For instance, Lee and Chan ${ }^{158}$ theoretically proposed that a chiral layerby-layer photonic crystal structure can thermally emit predominantly circularly polarized infrared light (around $12 \mu \mathrm{m}$ ), which arises from the polarization-dependent photonic band gaps or the surface plasmons if the photonic crystal structure is supported by a metallic substrate. Wu et al. ${ }^{159}$ proposed a silicon-based chiral metasurface in which the mirror symmetry is broken for a single meta-atom (like a broken " $n$ "-shape). It was shown that highly circularly polarized thermal radiation can be achieved in this structure at a wavelength about $4.7 \mu \mathrm{m}$. Notably, some metasurfaces that can introduce the spin-orbit interaction (SOI) of light, that is, the coupling of the photon spin (or circular polarization) and the extrinsic momentum (wavevector), can be engineered to thermally emit circularly polarized light. For instance, Shitrit et al. ${ }^{145}$ demonstrated that, by engineering the space-variant orientation angle for the nanoantennas in the metasurface, a photon-spin-dependent wavevector shift can be induced, which is called the optical Rashba effect as a manifestation of SOI under broken inversion symmetry. Therefore, the degenerate dispersion relations of different spins are split into two nondegenerate dispersions, leading to spin-dependent thermal emissivities, as demonstrated in Fig. 8(c), where $\sigma_{ \pm}= \pm 1$ indicates the photon spin corresponding to right and left polarization states of light, respectively. Note this behavior comes from the geometric phase generated by the entire system rather than the chiral response of individual meta-atoms as discussed in Section 5. Similar works include Refs. [124, 160]. Recently, Khandekar and $\mathrm{Jacob}^{161}$ theoretically predicted that circularly polarized emission can be generated by using a compact dimer of subwavelength anisotropic antennas when these antennas are under thermal nonequilibrium (i.e., different temperatures). It was further shown that the handedness of the emitted radiation is flipped upon interchanging the temperatures of the antennas.

Finally, let us discuss some works that can simultaneously realize multiple functions. As a matter of fact, MIM metamaterials and metasurfaces are widely used to realize thermal emitters which are narrowband and directive at the same time. For instance, Costantini et al. ${ }^{112}$ introduced a plasmonic MIM metastructure to control simultaneously the spectrum and the directivity of blackbody radiation, which can operate reliably at $600{ }^{\circ} \mathrm{C}$ with an emissivity higher than 0.85 in a narrow frequency band and in a narrow solid angle. It is already noted from the metamaterial section (Section 4) that hyperbolic metamaterials can also be designed to exhibit strong monochromatic and directional thermal emission simultaneously, for instance the work by Campione et $a l^{87}$ based on $\operatorname{In}_{0.53} \mathrm{Ga}_{0.47} \mathrm{As}$ and 8 -nm-thick undoped $\mathrm{Al}_{0.48} \mathrm{In}_{0.52} \mathrm{As}$ multilayers. Barbillon et al. ${ }^{162}$ showed some type-II hyperbolic materials that possess a Brewster angle can emit a highly 

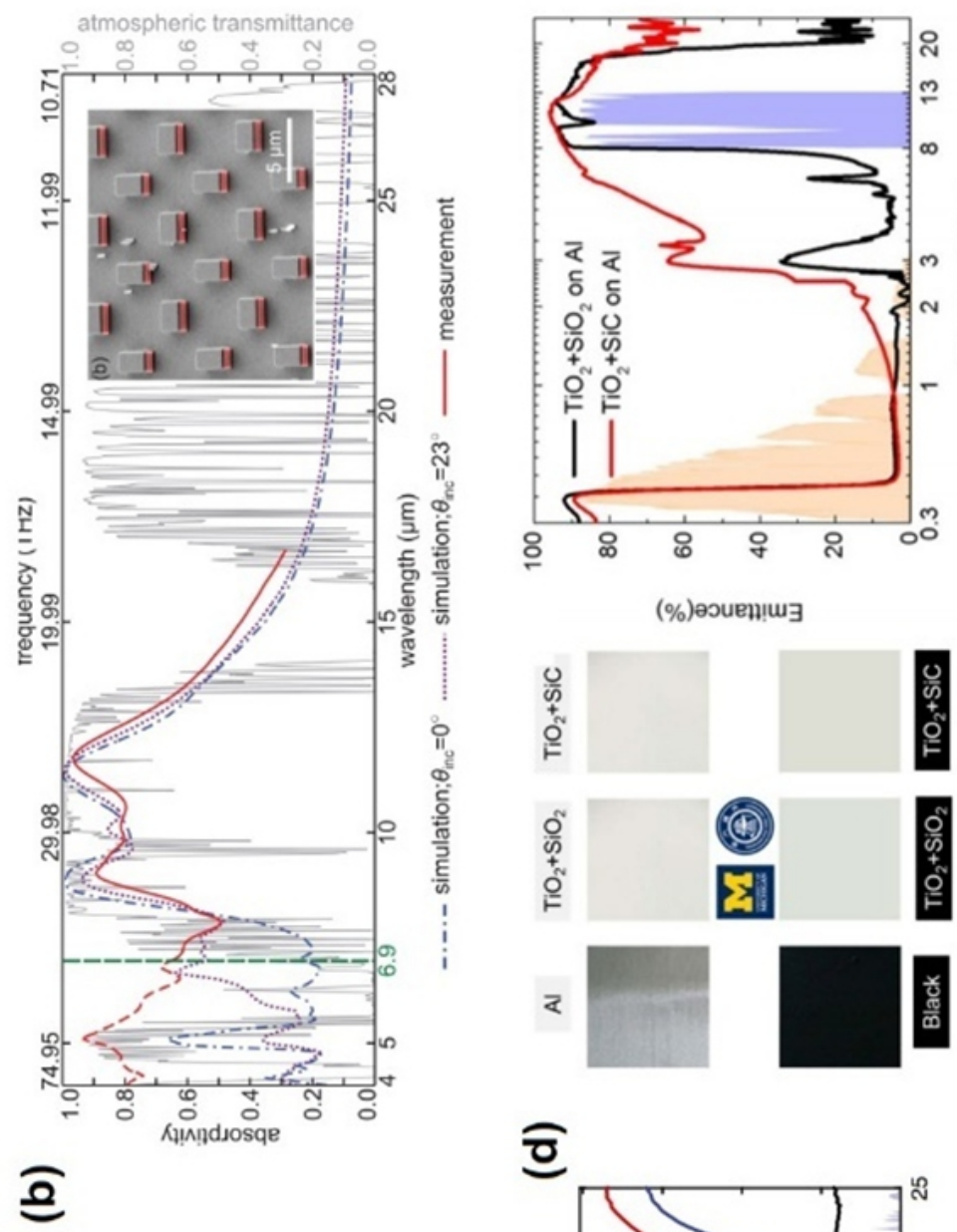

(\%)อวuอม!นษ

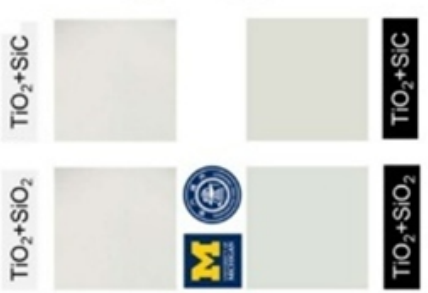

\&

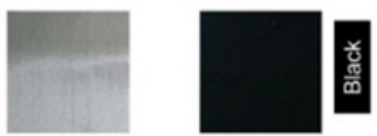

ㅎ
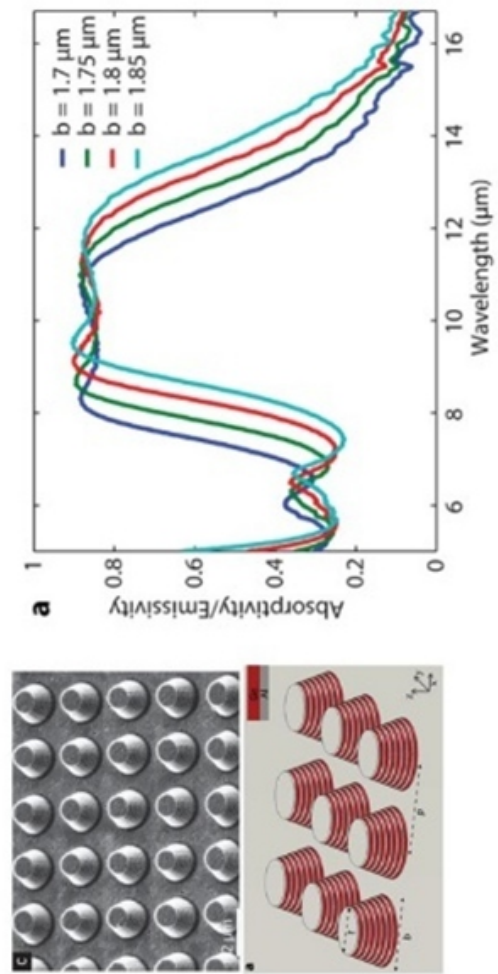

త్

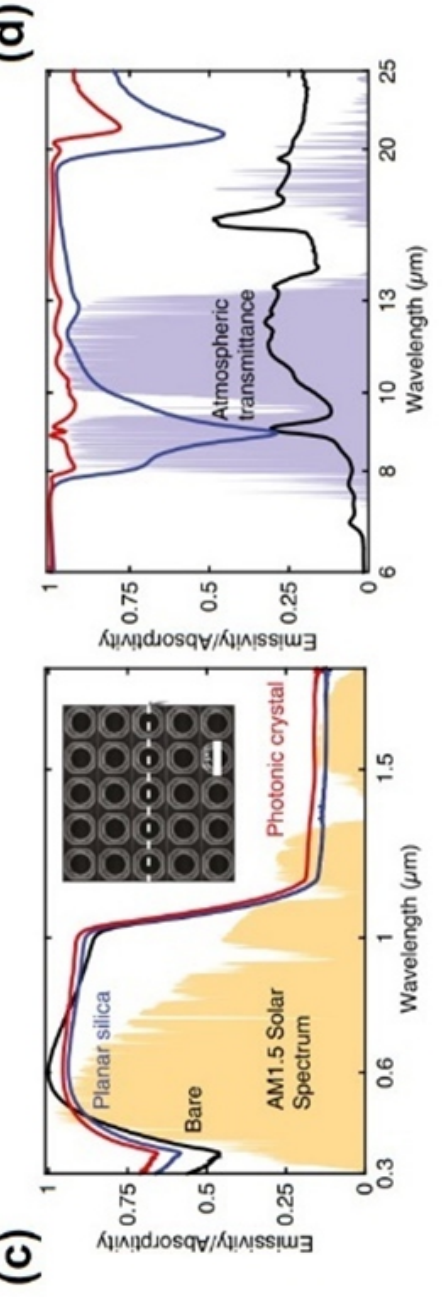

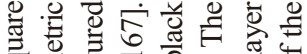
क्ष

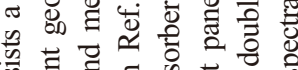
ใิ

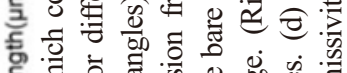

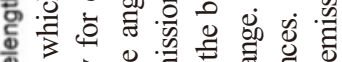

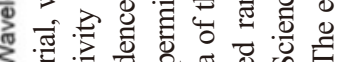

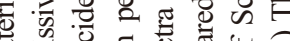

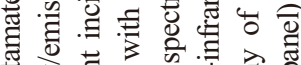

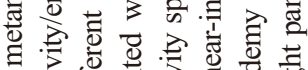

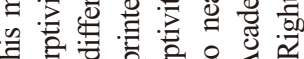

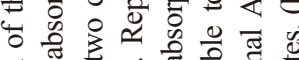

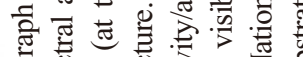

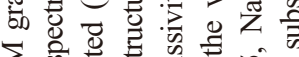

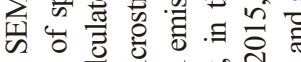

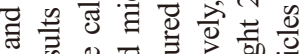
可 g च

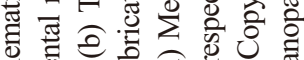

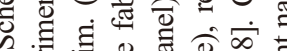
语言总高宫 窟

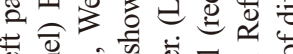

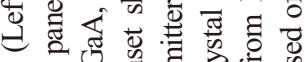

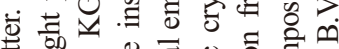

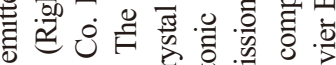

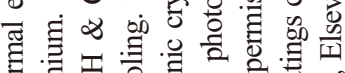

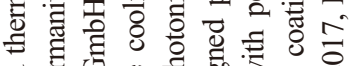
च है उ

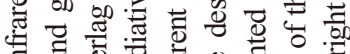

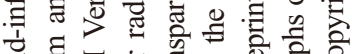

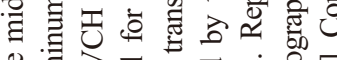

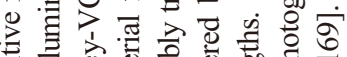

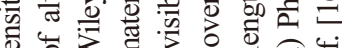

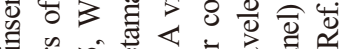

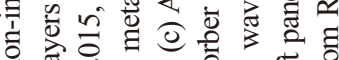

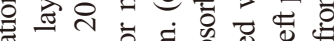

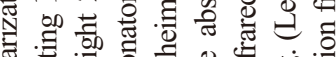

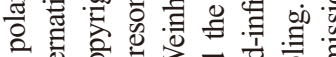

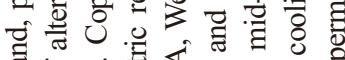
ठㄴ 尊 《完崩

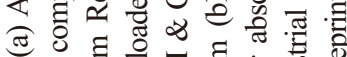

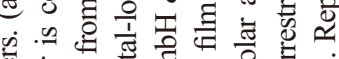

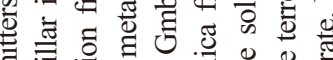

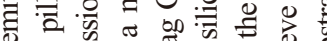

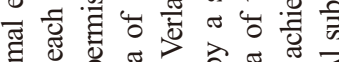

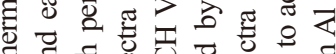

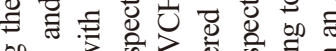
然娄 क人

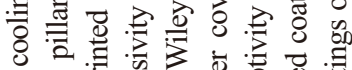

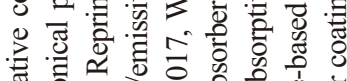
范

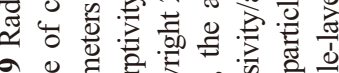

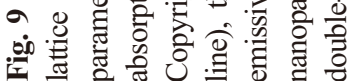


directional and $p$-polarized thermal radiation, and since the Brewster angle weakly depends on the wavelength, this emitter can work in a relatively wide spectral range. As previous mentioned, the $\mathrm{SiC}$ grating structure is a typical highly directional and polarized thermal light source. ${ }^{32}$ Dyakov et al. ${ }^{153}$ proposed that a slab ZnSe waveguide etched with chiral microstructures of fourfold rotational symmetry can thermally emit circularly polarized radiation in a narrowband, as presented in Fig. 8(d). Because of the circularly polarized eigenmodes supported by such metamaterial, it is seen that the degree of circular polarization (DCP) can be as high as 0.87 at $13 \mu \mathrm{m}$ for RCP and for LCP, the DCP reached -0.73 (not shown in the figure), and the narrowband emission arises from the Fano resonance nature of the quasi-guided modes which are the interaction of the discrete guided modes of the waveguide with the photon continuum in air. Notably, the work by Inoue et $a l^{147}$ presented a ultranarrowband $(Q>100)$ and highly directive (an angular range of $\sim 20^{\circ}$ near the normal direction) thermal emitter that radiates TM polarized waves at a wavelength of 9.1 $\mu \mathrm{m}$, which is an array of MQW rods that form a $2 \mathrm{D}$ photonic crystal slab.

\subsection{Daytime radiative cooling}

Recently, there has been growing interest in designing micro/ nanostructured thermal emitters that can efficiently achieve radiative cooling, that is, to utilize the capability of the Universe as cold sink (with a temperature around $3 \mathrm{~K}$ ) through the radiative energy transfer channel provided by the atmospheric window $(8-13 \mu \mathrm{m})$ to cool down terrestrial objects with a temperature around $300 \mathrm{~K}^{163}$ In this application, a highly emissive thermal radiator that work in the atmospheric transparency window is the key component, which directly determine the cooling power. Moreover, since for daytime terrestrial radiative cooling it is vital to minimize the heating effect induced by the absorption of solar irradiation, this kind of thermal emitter is required to be highly reflective in the visible to near-infrared wavelength range. Therefore, judiciously designed micro/nanostructures and appropriate materials should be employed to achieve the required highly wavelength-selective emissivity. In this subsection, we briefly introduce typical thermal emitters for radiative cooling, in which our focus is to analyze how the combination of various micro/nanostructures and materials can achieve the desired spectral properties. Other figure of merits like cooling power, temperature reduction and long-time performance will not be discussed in detail, since they are out of the scope of this review, see Ref.[164] for more details. A recent review on different micro/nanostructures can be found in Ref. [165].

Raman et al. ${ }^{170}$ introduced an integrated photonic solar reflector and thermal emitter consisting of seven alternating layers of $\mathrm{HfO}_{2}$ and $\mathrm{SiO}_{2}$, which can reflect $97 \%$ of the incident solar radiation and is highly emissive in the atmospheric transparency window. In this seven-layer structure, the top three layers with larger thicknesses are responsible for the high mid-infrared thermal emissivity due to the combination of phonon polaritonic resonance in $\mathrm{SiO}_{2}$ and the interference effects in the three layers. At the same time, the bottom four thin layers, forming a simple photonic crystal, are responsible for the high reflection in the solar spectrum. Hossain et al. ${ }^{166}$ demonstrated a radiative cooling thermal emitter based on an anisotropic and conical-shaped metamaterial structure, as shown in Fig. 9(a). This metamaterial consists a square lattice of conical pillars and each pillar is composed of alternating layers of aluminum ( $\mathrm{Al})$ and germanium $(\mathrm{Ge})$, where the thickness of the Al layer is $30 \mathrm{~nm}$ and that of the Ge layer is $110 \mathrm{~nm}$. Experimental results verified that this metamaterial showed a significantly high in the midinfrared atmospheric transparency window, as presented in Fig. 9(a). This broadband emissivity/absorptivity enhancement is attributed to the gradual absorption enhancement at different positions of the conical pillars for different wavelengths, arising from the flat dispersion induced low group velocities in the pillar waveguide, which was extensively discussed in similar metamaterial structures. ${ }^{171,}{ }^{172}$ Zou et al. ${ }^{167}$ devised a radiative cooling metamaterial composed of a $2 \mathrm{D}$ array of dielectric resonators made of doped silicon sandwiched between thin Ag layers on a doped silicon substrate. The calculated and measured absorptivity/emissivity spectra are presented in Fig. 9(b), where the inset shows the SEM graph of the fabricated microstructure. It was demonstrated that two magnetic resonances, one supported by the doped silicon resonator and another formed in the gaps between $\mathrm{Ag}$ layers, contribute to the high emissivity in the mid-infrared.

It should be noted that a different spectral response should be engineered for the radiative cooling emitters for solar absorbers and cells, which are required to be, on the other hand, transparent in the operating wavelength range of solar absorbers and cells. For instance, Zhu et al. ${ }^{168}$ proposed a silica photonic crystals emitter used to radiatively cool a solar absorber. It was demonstrated that the photonic crystal is visibly transparent and does not affect the absorption of the underneath absorber in the visible range, as shown in the left panel of Fig. 9(c), with a SEM graph of the photonic crystal. On the other hand, it is highly emissive in the mid-infrared as indicated in the right panel of Fig. 9(c).

Disordered micro/nanostructures are indeed promising in large scale radiative cooling. A notable work is the disordered metamaterial based on a polymeric thin film embedded with randomly distributed $\mathrm{SiO}_{2}$ microspheres by Zhai et al., ${ }^{14}$ as already discussed previously in Section 4. Recently, this type of metamaterial has been demonstrated to be capable of cooling down large thermal mass water to $10.6{ }^{\circ} \mathrm{C}$ below the ambient temperature around noon under direct sunlight. ${ }^{173}$ Bao et $a l .{ }^{169}$ also proposed a simple type of double-layer nanoparticle-based coatings to achieve terrestrial radiative cooling. This metamaterial coating is composed of a reflective $\mathrm{TiO}_{2}$ nanoparticle layer on top of a thermally emissive $\mathrm{SiC}$ (or $\mathrm{SiO}_{2}$ ) nanoparticle layer. Satisfactory wavelength-selective emissivity spectra are observed, as illustrated in Fig. 9(d). Moreover, Atiganyanun et $a l^{174}$ showed that a coating only made of randomly packed silica microspheres can simultaneously achieve a high thermal emissivity across the mid-infrared and a high reflection for solar irradiation if the size of the microspheres and the thickness of the coating are properly engineered. In a recent study, a kind of completely delignified and densified wood composed of cellulose fibers was demonstrated as scalable radiative cooling emitters. ${ }^{15}$ Recently, there have been a variety of works using simple two-layer or three-layer (bulk) structures to achieve daytime radiative cooling efficiently, which are low cost and lithography-free (even some are made of bulk materials), for example, Refs.[175-177].

\subsection{Thermophotovoltaics (TPV)}

Thermophotovoltaic systems aim to convert thermal energy into electricity by converting heat into electron-hole pairs in a low-bandgap photovoltaic medium, which have a great potential in remote power generation and waste heat harvesting. A conventional TPV system is commonly comprised of a thermal emitter, an optical filter (antireflection coating), and a TPV cell. When the thermal radiation provided by a broadband thermal emitter propagates through an optical filter, which reflects the non-convertible thermal energy, then a selective TPV emitter can be realized. ${ }^{178}$

On the other hand, the conversion efficiency of TPV can be further improved by applying selective emitters based on PCs and metamaterials. Nagpa et al. ${ }^{179}$ employed the directive laser writing technique to fabricate a 3D molybdenum woodpile PC thermal emitter, 

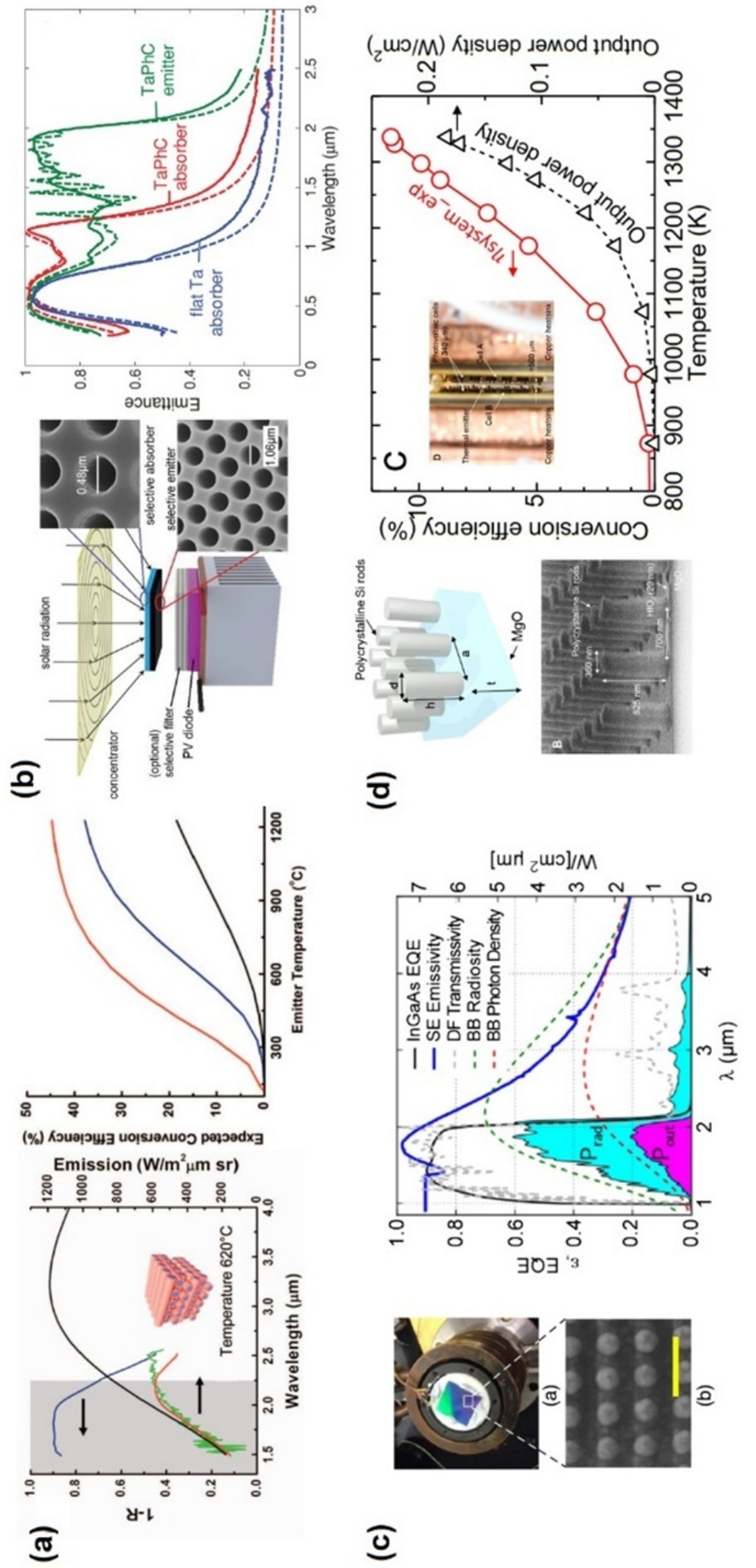

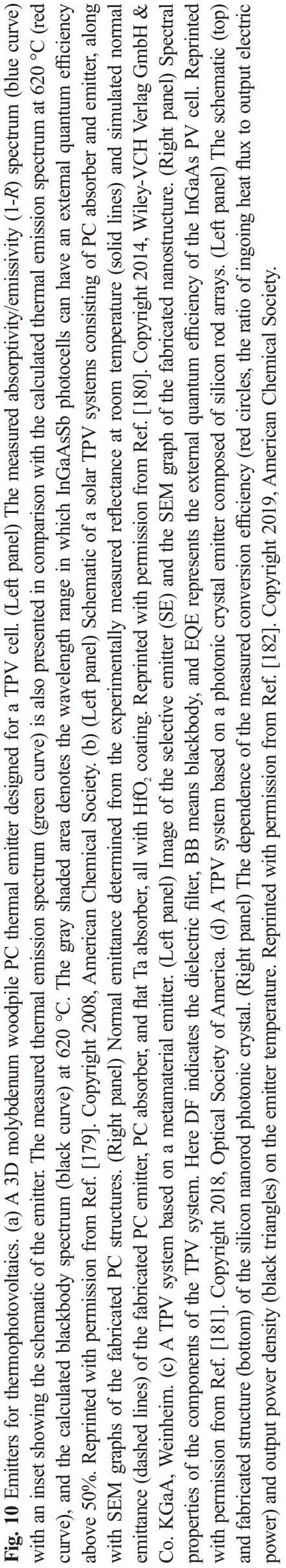


whose experimentally measured emissivity and emission power spectra are shown in the left panel of Fig. 10(a). The right panel of Fig. 10(a) gives the calculated results of the optical-to-electrical conversion efficiency for the TPV cell as a function of the operating temperature if a narrow band gap InGaAsSb PV cell is applied. It is found that the optical-to-electrical conversion efficiency can be above $32 \%$ at a typical operating temperature of $650{ }^{\circ} \mathrm{C}$. Rinnerbauer et al. ${ }^{180}$ demonstrated a 2D PC thermal absorber/emitter pair comprising a square lattice of cylindrical cavities etched into a Ta substrate and achieve a measured system efficiency of $3.74 \%$ at a realistic target operating temperature of $1300 \mathrm{~K}$, which exhibited more than twice the performance of nearblackbody absorber at the solar irradiance of $130 \mathrm{~kW} \mathrm{~m}^{-2}$. The schematic and experimentally determined emittance spectra are presented in Fig.10(b). Furthermore, Lenert et al. ${ }^{11}$ designed a complete high-efficiency solar TPV (STPV) device with an experimental efficiency of $3.2 \%$, in which $\mathrm{Si} / \mathrm{SiO}_{2}$ multilayer PC structure was fabricated as the selective thermal emitter. The emission spectrum was designed to match the band gap of the PV cell (0.55 eV-bandgap InGaAsSb PV cell), crucial to the relatively high conversion efficiency. Woolf et al. ${ }^{181}$ experimentally demonstrated the first metamaterial selective emitter for TPV. The thermal emitter consisted of an array of platinum pucks separated from a platinum backplane by the $\mathrm{Al}_{2} \mathrm{O}_{3}$ layer, whose SEM graph is given in Fig. 10(c). It was demonstrated that this system has a thermal-to-electrical power conversion efficiency of $24.1 \pm 0.9 \%$ at an operating temperature of $1055^{\circ} \mathrm{C}$, in which a $0.6 \mathrm{eV}$ band gap $\mathrm{In}_{0.68} \mathrm{Ga}_{0.32}$ As PV cell was used, and the spectral properties of the components are shown in the right panel of Fig. 10(c). Recently, Suemitsu et al. ${ }^{182}$ experimentally investigated a TPV system consisting of a silicon rod photonic crystal as thermal emitter (see Fig. 10(d)) with a relatively narrow emission spectrum and a photovoltaic cell with a band gap corresponding to $1.76 \mu \mathrm{m}$ (InGaAs PV cell). The power generation efficiency was also measured, reaching $11.2 \%$ at an operating temperature of $1338{ }^{\circ} \mathrm{C}$, as presented in the right panel of Fig. 10(d). They further discussed that if excluding the losses that can be reduced via upscaling, the system efficiency of $14.5 \%$ could be obtained, and by further improving the contact grid design of the PV cell an even higher efficiency about $19.4 \%$ could be expected.

\subsection{Thermal camouflage}

Thermal camouflage, which possesses the ability to disguise and hide the object in the background based on their radiation difference, has attracted growing attention recently. In general, there are two types thermal-controlled methods to design thermal camouflage devices, including conduction-based thermal camouflage and radiation-based thermal camouflage. Usually, the realization of conduction-typed camouflage is on the base of transformation thermotics. ${ }^{183-186}$ In this review, we mainly focus on the recent progress of radiation-typed camouflage.

By tailoring thermal emissivity of encapsulated materials, it is possible to disguise the infrared detectors. This thermal camouflage is achieved mainly by reducing thermal emissivity to match the background. For example, in Fig. 11(a), Lee et l. $^{20}$ proposed MIMbased emitter to achieve infrared camouflage, which possesses a low emissivity in atmospheric windows (detected bands) $(3 \sim 5 \mu \mathrm{m}$ and $8 \sim$ $14 \mu \mathrm{m})$ and high emissivity in undetected band $(5-8 \mu \mathrm{m})$. For a targeted temperature, the desirable performance can be realized by changing the radius of nanodisks. Recently, many researchers tend to combine active materials to achieve tunable thermal camouflage. For instance, $\mathrm{Qu}$ et $a l^{21}$ have successfully demonstrated dynamically thermal camouflage by employing phasing-changing materials GST as shown in Fig. 11(b). Near perfect camouflage function can be maintained covering a broad background temperature $\left(30{ }^{\circ} \mathrm{C}-50{ }^{\circ} \mathrm{C}\right)$ and a wide range of observation angle $\left(0-60^{\circ}\right)$, which indeed stimulates a new branch of thermal camouflage technology. Besides,
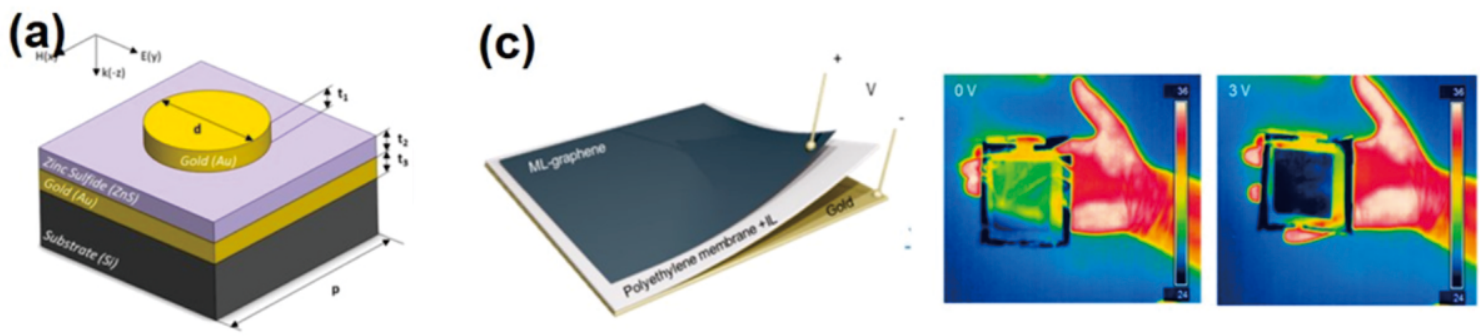

(b)

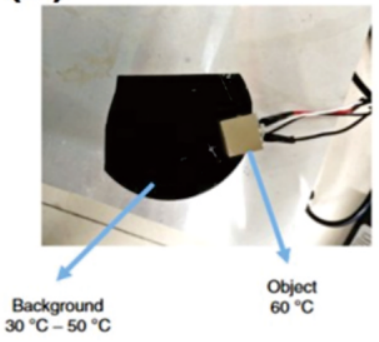

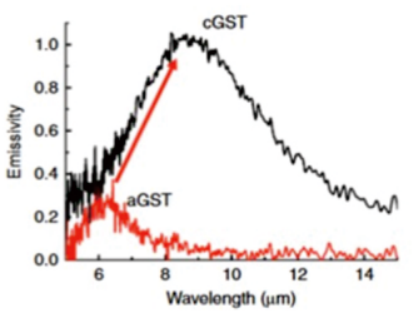

(d)

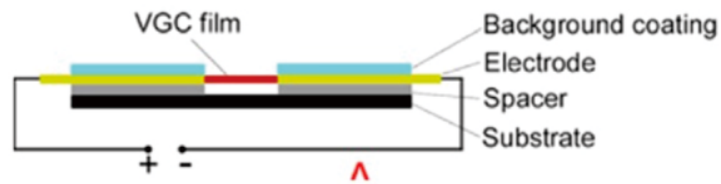

Fig. 11 Thermal camouflage. (a) Schematic of the unit cell of thermal emitter for thermal camouflage in Ref. ${ }^{20}$. Reprinted with permission from Ref. [20]. Copyright 2019, American Chemical Society. (b) The experimental setup for GST-based thermal camouflage (left) and emissivity spectra of GST material at two phase (right). Reprinted with permission from Ref. [21] under the terms of the Creative Commons Attribution 4.0 International License. (c) Schematic of thermal surface composed of multilayer-graphene electrode, a porous polyethylene membrane and a black gold-electrode coated on heat resistive nylon. The two right figures show thermal camera images of designed device under the voltage bias of 0 and $3 \mathrm{~V}$. Reprinted with permission from Ref. [187]. Copyright 2018, American Chemical Society. (d) Schematic of the adaptive thermal camouflage. Reprinted from Ref. [188]. Copyright 2015, American Chemical Society. 
graphene-based thermal camouflage devices (see Fig. 11(c)) has also been designed by introducing intercalation of a nonvolatile ionic liquid into graphene layers using porous substrate. ${ }^{187}$ This type of tunable camouflage can work at full mid-infrared region, and electrical control of thermal emissivity $(0.3-0.8)$ can be achieved in real-time, exhibiting outstand adaptive camouflage functions by disguising hot surface as cold one and vice versa. Furthermore, in Fig. 11(d), Xiao et al. ${ }^{188}$ demonstrated a $\mathrm{VO}_{2}$ /graphene/CNT (carbon nanotube) metasurfaces to achieve adaptive thermal camouflage, showing large tunability in engineering thermal emissivity. Specifically, the $\mathrm{VO}_{2}$ phase transition can be dynamically driven by graphene/CNT, which has been designed to achieve dynamical manipulation of thermal radiation of objects being similar to the background. This thermal camouflage metadevice exhibits a switchable response, lower power consumption and high stability, which can also be applied to diverse substrates.

\section{Summary and Outlook}

In this paper, we give an overview of micro/nanostructures to control far-field thermal emission. Various thermal emitters based on different micro/nanostructures including gratings, photonic crystals, metamaterials and metasurfaces are reviewed and some important applications based on them, such as directive, narrowband and polarized thermal light sources, thermophotovoltaics, thermal camouflage and radiative cooling, are also summarized. We also discuss some works that have realized

Table 1 Typical classification of different micro/nanostructures.

\begin{tabular}{|c|c|c|c|}
\hline & $1 \mathrm{D}$ & $2 \mathrm{D}$ & $3 \mathrm{D}$ \\
\hline \multirow[t]{4}{*}{ Grating } & $\bar{d}_{x} \sim 1$ & $\bar{d}_{x} \sim 1$ & $\bar{d}_{x} \sim 1$ \\
\hline & $\bar{d}_{y} \rightarrow \infty$ & $\bar{d}_{y} \sim 1$ & $\bar{d}_{y} \sim 1$ \\
\hline & $\bar{d}_{z} \rightarrow \infty$ & $\bar{d}_{z} \rightarrow \infty$ & $\bar{d}_{z} \sim 1$ \\
\hline & $\forall \bar{L}_{z}$ & $\forall \bar{L}_{z}$ & $\bar{L}_{z} \gg \bar{d}_{z}$ \\
\hline \multirow[t]{4}{*}{ Photonic Crystal } & $\bar{d}_{x} \rightarrow \infty$ & $\bar{d}_{x} \sim 1$ & $\bar{d}_{x} \sim 1$ \\
\hline & $\bar{d}_{y} \rightarrow \infty$ & $\bar{d}_{y} \sim 1$ & $\bar{d}_{y} \sim 1$ \\
\hline & $\bar{d}_{z} \sim 1$ & $\bar{d}_{z} \rightarrow \infty$ & $\bar{d}_{z} \sim 1$ \\
\hline & $\bar{L}_{z} \gg \bar{d}_{z}$ & $\forall \bar{L}_{z}$ & $\bar{L}_{z} \gg \bar{d}_{z}$ \\
\hline \multirow[t]{4}{*}{ Metamaterial } & $\bar{d}_{x} \rightarrow \infty$ & $\bar{d}_{x} \ll 1$ & $\bar{d}_{x} \ll 1$ \\
\hline & $\bar{d}_{y} \rightarrow \infty$ & $\bar{d}_{y} \ll 1$ & $\bar{d}_{y} \ll 1$ \\
\hline & $\bar{d}_{z} \ll 1$ & $\bar{d}_{z} \rightarrow \infty$ & $\bar{d}_{z} \ll 1$ \\
\hline & $\bar{L}_{z} \gg \bar{d}_{z}$ & $\forall \bar{L}_{z}$ & $\bar{L}_{z} \gg \bar{d}_{z}$ \\
\hline \multirow[t]{4}{*}{ Metasurface } & $\bar{d}_{x} \ll 1$ & $\bar{d}_{x} \ll 1$ & $\bar{d}_{x} \ll 1$ \\
\hline & $\bar{d}_{y} \rightarrow \infty$ & $\bar{d}_{y} \ll 1$ & $\bar{d}_{y} \ll 1$ \\
\hline & $\bar{d}_{z} \rightarrow \infty$ & $\bar{d}_{z} \rightarrow \infty$ & $\bar{d}_{z} \ll 1$ \\
\hline & $\bar{L}_{z} \ll 1$ & $\bar{L}_{z} \ll 1$ & $\bar{L}_{z} \ll 1 *$ \\
\hline
\end{tabular}

*Here 3D metasurface is equivalent to the so-called few-layer metasurface that consists of several cascaded metasurfaces. 
reconfigurable thermal emission.

This paper is not intended to be an exhaustive review and it is also not possible for us to make it exhaustive because there are so many excellent works in the last several decades trying to tailor far-field thermal emission using different micro/nanostructures and materials based on different design principles. In the following, we try to present some very personal perspectives.

From a fundamental point of view, following the line of the development of micro/nanostructures, metasurfaces are, to some extent, the most promising structures to achieve versatile, multifunctional and efficient manipulation of far-field emission. More importantly, they can be more easily integrated with each other or into other nanodevices than other types of micro/nanostructures. In this sense, we can say that metasurfaces are the "structures of the future generation". On the other hand, from a practical point of view, different structures have their own advantages and should be carefully chosen according to the requirements of concrete applications. And in particular, it is very much inspiring to see that some disordered metamaterials have been successfully demonstrated to be put forward to large-scale practical applications, like the radiative cooling metamaterial coatings. ${ }^{173}$

From previous discussions, we can see that the study of controlling thermal radiation has benefited a lot from the rapid developments of nanophotonics and nanooptics. In this respect, we also envisage that the prosperity of topological photonics, ${ }^{189}$ non-Hermitian optics $^{190}$ and quantum nanophotonics ${ }^{191}$ in recent years will bring new concepts to the micro/nanoscale control of thermal radiation. For example, the connection between topological photonics and thermal radiation has been made theoretically by a recent work of Silveirinha. ${ }^{192}$ Non-Hermitian selective thermal emitters exhibiting passive PTsymmetry at $700{ }^{\circ} \mathrm{C}$ were very recently demonstrated by Doiron and Naik, ${ }^{193}$ in which a PT phase transition makes the dual emission peaks converges into a single emission peak in the parameter space. Ridolfo et $a l .^{194}$ theoretically showed that the photon statistics of thermal radiation emitted from a cavity quantum electrodynamics (QED) system in the ultrastrong coupling regime vastly differs from conventional statistics of thermal photons, which implies a possible way to tailor the photon statistics of thermal radiation using quantum nanophotonic tools.

On the other hand, the discovery of novel nano- and quantum materials, like graphene, transition metal dichalcogenides (TMDs), ${ }^{195}$ black phosphorus, ${ }^{196}$ other 2D materials and their heterostructures, ${ }^{197,198}$ as well as topological insulators and superconductors, ${ }^{199}$ drastically expands the fundamental limits of our scope into the extreme nanoscale (even the atomistic scale). The tunability of their electronic and optical properties is impressive. The unconventional and rich optical phenomena in them can offer a promising platform for realizing exotic thermal radiation phenomena and achieving high thermal radiation emission performance. ${ }^{200,}{ }^{201}$ One possible idea is to thermally excite topologically protected edge currents in topological insulators to achieve narrowband thermal emission that is protected and very robust over imperfections and disorder. This is helpful for novel thermal radiation sources and detectors. In practice, there are still many physical mechanisms not fully explored, and the nanofabrication, compatible integration and high-precision measurement remain demanding. Nevertheless, we are still looking forward to the applications of these interesting materials in manipulating far-field thermal emission.

\section{Conflict of interest}

There are no conflicts to declare.

\section{Acknowledgements}

We thank the financial support from the National Natural Science Foundation of China (No. 51636004 and No. 51906144), Shanghai Key Fundamental Research Grant (No. 18JC1413300), China Postdoctoral Science Foundation (No. BX20180187 and No. 2019M651493) and the Foundation for Innovative Research Groups of the National Natural Science Foundation of China (No. 51521004).

\section{Appendix}

As a summary of the discussion in the maintext, here in Table I, we present a simple classification of typical micro/nanostructures for thermal emitters based on the length scale at different dimensions with respect to the radiation wavelength of interest to aid the readers. Here, without loss of generality, $\bar{d}_{x}=d_{x} / \lambda, \bar{d}_{y}=d_{y} / \lambda, \bar{d}_{z}=d_{z} / \lambda$ are normalized characteristic length of micro/nanostructures with respect to the wavelength $\lambda$. The Cartesian coordinates are chosen such that $z$ indicates the dimension of thickness (aligned with normal emission/incidence direction) and $x, y$ are in-plane dimensions. We use $\bar{L}_{z}=L_{z} / \lambda$ to denote the normalized thickness of the emitter. Here $\bar{d} \rightarrow \infty$ means infinitely large (namely, no patterned micro/nanostructures) and $\forall L$ means the length is arbitrary (but not infinitely large or small in practice).

\section{References}

1. J. R. Howell, M. P. Menguc and R. Siegel, Therm. Radiat. Heat Tran., CRC press, 2015.

2. J. Yao, Z. Liu, Y. Liu, Y. Wang, C. Sun, G. Bartal, A. M. Stacy and X. Zhang, Science, 2008, 321, 930.

3. S. Xi, H. Chen, T. Jiang, L. Ran, J. Huangfu, B. I. Wu, J. A. Kong and M. Chen, Phys. Rev. Lett., 2009, 103, 194801.

4. A. Grbic, L. Jiang and R. Merlin, Science, 2008, 320, 511.

5. W. Cai, U. K. Chettiar, A. V. Kildishev and V. M. Shalaev, Nat. Photonics, 2007, 1, 224-227.

6. T. Baba, Nat. Photonics, 2008, 2, 465-473.

7. S. Fan, Joule, 2017, 1, 264-273.

8. W. Li and S. Fan, Opt. Express, 2018, 26, 15995-16021.

9. B. Zhao and Z. M. Zhang, in Handbook of Thermal Science and Engineering, Springer International Publishing, Cham, 2018, pp. 1023-1068.

10. D. G. Baranov, Y. Xiao, I. A. Nechepurenko, A. Krasnok, A. Alù and M. A. Kats, Nat. Mater., 2019, 18, 920-930.

11. A. Lenert, D. M. Bierman, Y. Nam, W. R. Chan, I. Celanović, M. Soljačić and E. N. Wang, Nat. Nanotechnol., 2014, 9, 126.

12. Z. Zhou, E. Sakr, Y. Sun and P. Bermel, in Nanophotonics, 2016, p. 1.

13. C. Wu, B. Neuner Iii, J. John, A. Milder, B. Zollars, S. Savoy and G. Shvets, J. Optics-UK, 2012, 14, 024005.

14. Y. Zhai, Y. Ma, S. N. David, D. Zhao, R. Lou, G. Tan, R. Yang and X. Yin, Science, 2017, 355, 1062

15. T. Li, Y. Zhai, S. He, W. Gan, Z. Wei, M. Heidarinejad, D. Dalgo, R. Mi, X. Zhao, J. Song, J. Dai, C. Chen, A. Aili, A. Vellore, A. Martini, R. Yang, J. Srebric, X. Yin and L. Hu, Science, 2019, 364, 760.

16. A. Lochbaum, Y. Fedoryshyn, A. Dorodnyy, U. Koch, C. Hafner and J. Leuthold, ACS Photonics, 2017, 4, 1371-1380.

17. G. Pühringer and B. Jakoby, Materials, 2019, 12.

18. A. Pusch, A. De Luca, S. S. Oh, S. Wuestner, T. Roschuk, Y. Chen, S. Boual, Z. Ali, C. C. Phillips, M. Hong, S. A. Maier, F. Udrea, R. H. Hopper and O. Hess, Sci. Rep-UK, 2015, 5, 17451.

19. O. Ilic, P. Bermel, G. Chen, J. D. Joannopoulos, I. Celanovic and M. Soljačić, Nat. Nanotechnol., 2016, 11, 320-324.

20. N. Lee, T. Kim, J. S. Lim, I. Chang and H. H. Cho, ACS Appl. Mater. Inter. 2019, 11, 21250-21257.

21. Y. Qu, Q. Li, L. Cai, M. Pan, P. Ghosh, K. Du and M. Qiu, Light-Sci. Appl., 2018, 7, 26.

22. X. Liu, L. Wang and Z. M. Zhang, Nanosc. Microsc. Therm., 2015, 19, 98126.

23. S. Basu, Z. M. Zhang and C. J. Fu, Int. J. Energ. Res., 2009, 33, 1203-1232.

24. E. Tervo, E. Bagherisereshki and Z. Zhang, Frontiers in Energy, 2018, 12, 5-21. 
25. B. Song, A. Fiorino, E. Meyhofer and P. Reddy, AIP Adv, 2015, 5, 053503.

26. J. W. Yoon, K. J. Lee, W. Wu and R. Magnusson, Adv. Opt. Mater, 2014, 2 , 1206-1212.

27. J. Le Gall, M. Olivier and J. J. Greffet, Phys. Rev. B, 1997, 55, 10105-10114.

28. P. J. Hesketh, J. N. Zemel and B. Gebhart, Phys. Rev. B, 1988, 37, 1079510802 .

29. P. J. Hesketh, J. N. Zemel and B. Gebhart, Phys. Rev. B, 1988, 37, 1080310813.

30. T. K. Wang and J. N. Zemel, Infrared Phys., 1991, 32, 477-488.

31. P. J. Hesketh, J. N. Zemel and B. Gebhart, Nature, 1986, 324, 549-551.

32. J. J. Greffet, R. Carminati, K. Joulain, J. P. Mulet, S. Mainguy and Y. Chen, Nature, 2002, 416, 61-64.

33. C. Fu and Z. M. Zhang, Frontiers of Energy and Power Engineering in China, 2009, 3, 11-26.

34. I. E. Khodasevych, L. Wang, A. Mitchell and G. Rosengarten, Adv. Opt. Mater., 2015, 3, 852-881.

35. J. Liu, U. Guler, A. Lagutchev, A. Kildishev, O. Malis, A. Boltasseva and V. M. Shalaev, Opt. Mater. Express, 2015, 5, 2721-2728.

36. Y. B. Chen and Z. M. Zhang, Opt. Commun., 2007, 269, 411-417.

37. N. Dahan, A. Niv, G. Biener, Y. Gorodetski, V. Kleiner and E. Hasman, Phys. Rev. B, 2007, 76, 045427 .

38. D. Crouse, E. Jaquay, A. Maikal and A. P. Hibbins, Phys. Rev. B, 2008, 77, 195437.

39. Y. Lu, M. H. Cho, Y. Lee and J. Y. Rhee, Appl. Phys. Lett., 2008, 93, 061102.

40. N. Nguyen-Huu, Y. B. Chen and Y. L. Lo, Opt. Express, 2012, 20, $5882-$ 5890.

41. C. Arnold, F. Marquier, M. Garin, F. Pardo, S. Collin, N. Bardou, J. L. Pelouard and J. J. Greffet, Phys. Rev. B, 2012, 86, 035316.

42. B. Zhao, J. M. Zhao and Z. M. Zhang, Appl. Phys. Lett., 2014, 105, 031905.

43. A. Heinzel, V. Boerner, A. Gombert, B. Bläsi, V. Wittwer and J. Luther, J. Mod. Optic., 2000, 47, 2399-2419.

44. A. Kohiyama, M. Shimizu, F. Iguchi and H. Yugami, J. Appl. Phys., 2015, 118, 133102

45. H. Sai, Y. Kanamori and H. Yugami, J. Micromech. Microeng., 2005, 15, S243-S249.

46. S. Maruyama, T. Kashiwa, H. Yugami and M. Esashi, Appl. Phys. Lett., 2001, 79, 1393-1395.

47. J. Song, H. Wu, Q. Cheng and J. Zhao, Journal of Quantitative Spectroscopy and Radiative Transfer, 2015, 158, 136-144.

48. L. P. Wang and Z. M. Zhang, Appl. Phys. Lett., 2009, 95, 111904.

49. B. J. Lee, L. P. Wang and Z. M. Zhang, Opt. Express, 2008, 16, 1132811336.

50. Y. Yang and L. Wang, Phys. Rev. Lett., 2016, 117, 044301.

51. H. Liu, D. A. Genov, D. M. Wu, Y. M. Liu, J. M. Steele, C. Sun, S. N. Zhu and X. Zhang, Phys. Rev. Lett., 2006, 97, 243902.

52. H. Liu, T. Li, Q. J. Wang, Z. H. Zhu, S. M. Wang, J. Q. Li, S. N. Zhu, Y. Y. Zhu and X. Zhang, Phys. Rev. B, 2009, 79, 024304.

53. T. Li, S. M. Wang, H. Liu, J. Q. Li, F. M. Wang, S. N. Zhu and X. Zhang, J. Appl. Phys., 2008, 103, 023104.

54. B. Zhao, Y. Shi, J. Wang, Z. Zhao, N. Zhao and S. Fan, Opt. Lett., 2019, 44, 4203-4206

55. J. D. Joannopoulos, S. G. Johnson, J. N. Winn and R. D. Meade, Photonic crystals: molding the flow of light, Princeton Univ. Press, Princeton, NJ, 2008.

56. A. Narayanaswamy and G. Chen, Phys. Rev. B, 2004, 70, 125101.

57. B. J. Lee, C. J. Fu and Z. M. Zhang, Appl. Phys. Lett., 2005, 87, 071904

58. M. U. Pralle, N. Moelders, M. P. McNeal, I. Puscasu, A. C. Greenwald, J. T. Daly, E. A. Johnson, T. George, D. S. Choi, I. El-Kady and R. Biswas, Appl. Phys. Lett., 2002, 81, 4685-4687.

59. H. Sai, H. Yugami, Y. Akiyama, Y. Kanamori and K. Hane, J. Opt. Soc. Am. A, 2001, 18, 1471-1476.

60. S. Enoch, J. J. Simon, L. Escoubas, Z. Elalmy, F. Lemarquis, P. Torchio and G. Albrand, Appl. Phys. Lett., 2005, 86, 261101.

61. M. Laroche, R. Carminati and J. J. Greffet, Phys. Rev. Lett., 2006, 96, 123903.

62. M. Ghebrebrhan, P. Bermel, Y. X. Yeng, I. Celanovic, M. Soljačić and J. D. Joannopoulos, Phys. Rev. A, 2011, 83, 033810.

63. S. Y. Lin, J. Moreno and J. Fleming, Appl. Phys. Lett., 2003, 83, 380-382.
64. K. A. Arpin, M. D. Losego, A. N. Cloud, H. Ning, J. Mallek, N. P. Sergeant, L. Zhu, Z. Yu, B. Kalanyan, G. N. Parsons, G. S. Girolami, J. R. Abelson, S. Fan and P. V. Braun, Nat. Commun., 2013, 4, 2630.

65. J. G. Fleming, S. Y. Lin, I. El-Kady, R. Biswas and K. M. Ho, Nature, 2002, 417, 52-55.

66. S. Y. Lin, J. G. Fleming and I. El-Kady, Opt. Lett., 2003, 28, 1909-1911.

67. S. E. Han, A. Stein and D. J. Norris, Phys. Rev. Lett., 2007, 99, 053906.

68. K. A. Arpin, M. D. Losego, A. N. Cloud, H. Ning, J. Mallek, N. P. Sergeant, L. Zhu, Z. Yu, B. Kalanyan, G. N. Parsons, G. S. Girolami, J. R. Abelson, S. Fan and P. V. Braun, Nat. Commun., 2013, 4, 2630.

69. D. Lu, J. J. Kan, E. E. Fullerton and Z. Liu, Nat. Nanotechnol., 2014, 9, 4853.

70. D. A. Pawlak, Scientia Plena, 2008, 4.

71. F. Lemoult, N. Kaina, M. Fink and G. Lerosey, Nat. Phys., 2013, 9, 55.

72. C. Sugino, S. Leadenham, M. Ruzzene and A. Erturk, J. Appl. Phys., 2016, 120, 134501.

73. M. V. Rybin, D. S. Filonov, K. B. Samusev, P. A. Belov, Y. S. Kivshar and M. F. Limonov, Nat. commun., 2015, 6, 10102.

74. X. Liu, T. Tyler, T. Starr, A. F. Starr, N. M. Jokerst and W. J. Padilla, Phys. Rev. Lett., 2011, 107, 045901.

75. X. Liu, T. Starr, A. F. Starr and W. J. Padilla, Phys. Rev. Lett., 2010, 104, 207403.

76. R. Adato, A. Artar, S. Erramilli and H. Altug, Nano Lett., 2013, 13, 25842591.

77. Y. Matsuno and A. Sakurai, Opt. Mater. Express, 2017, 7, 618-626.

78. T. Yokoyama, T. D. Dao, K. Chen, S. Ishii, R. P. Sugavaneshwar, M. Kitajima and T. Nagao, Adv. Opt. Mater., 2016, 4, 1987-1992.

79. Y. Gong, Z. Wang, K. Li, L. Uggalla, J. Huang, N. Copner, Y. Zhou, D. Qiao and J. Zhu, Opt. Lett., 2017, 42, 4537-4540.

80. T. Wang, P. Li, D. N. Chigrin, A. J. Giles, F. J. Bezares, O. J. Glembocki, J. D. Caldwell and T. Taubner, ACS Photonics, 2017, 4, 1753-1760.

81. X. Niu, X. Hu, S. Chu and Q. Gong, Adv. Opt. Mater., 2018, 6, 1701292.

82. I. Liberal and N. Engheta, P. Natl. Acad. Sci., 2018, 115, 2878.

83. S. Molesky, C. J. Dewalt and Z. Jacob, Opt. Express, 2013, 21, A96-A110.

84. P. N. Dyachenko, S. Molesky, A. Y. Petrov, M. Störmer, T. Krekeler, S. Lang, M. Ritter, Z. Jacob and M. Eich, Nat. Commun., 2016, 7, 11809.

85. Y. H. Kan, C. Y. Zhao, X. Fang and B. X. Wang, Opt. Lett., 2017, 42, 18791882.

86. T. I. Lee, J. W. Cho, D. Jeong, K. J. Lee, B. Y. Park, T. J. Kim, Y. D. Kim, Y S. Kim, Y. Nam and S. K. Kim, Phys. Status Solidi (a), 2018, 215, 1800287.

87. S. Campione, F. Marquier, J. P. Hugonin, A. R. Ellis, J. F. Klem, M. B. Sinclair and T. S. Luk, Sci. Rep-UK, 2016, 6, 34746.

88. I. S. Nefedov and L. A. Melnikov, Appl. Phys. Lett., 2014, 105, 161902.

89. S. A. Biehs and P. Ben-Abdallah, Phys. Rev. B, 2016, 93, 165405.

90. P. Sohr, C. I. Ip and S. Law, Opt. Lett., 2019, 44, 1138-1141.

91. A. Ghanekar, L. Lin and Y. Zheng, Opt. Express, 2016, 24, A868-A877.

92. A. Lagendijk, B. Nienhuis, B. A. van Tiggelen and P. de Vries, Phys. Rev. Lett., 1997, 79, 657-660.

93. T. Asano, M. Suemitsu, K. Hashimoto, M. De Zoysa, T. Shibahara, T. Tsutsumi and S. Noda, Sci. Adv., 2016, 2, e1600499.

94. J. Kong, T. Sun, F. Cao, Y. Li, J. Bao, J. Gao, X. Wang, G. Zhou, C. Guo, Z. Ren and K. Kempa, Phys. Status Solidi (a), 2018, 215, 1800206.

95. A. Sakurai, K. Yada, T. Simomura, S. Ju, M. Kashiwagi, H. Okada, T. Nagao, K. Tsuda and J. Shiomi, ACS Central Sci., 2019, 5, 319-326.

96. Y. Peng, J. Chen, A. Y. Song, P. B. Catrysse, P. C. Hsu, L. Cai, B. Liu, Y. Zhu, G. Zhou, D. S. Wu, H. R. Lee, S. Fan and Y. Cui, Nat. Sustainability, 2018, 1, 105-112.

97. P. C. Hsu, A. Y. Song, P. B. Catrysse, C. Liu, Y. Peng, J. Xie, S. Fan and Y. Cui, Science, 2016, 353, 1019.

98. J. Mandal, Y. Fu, A. C. Overvig, M. Jia, K. Sun, N. N. Shi, H. Zhou, X Xiao, N. Yu and Y. Yang, Science, 2018, 362, 315.

99. M. A. Kats, R. Blanchard, S. Zhang, P. Genevet, C. Ko, S. Ramanathan and F. Capasso, Phys. Rev. X, 2013, 3, 041004.

100. N. Yu and F. Capasso, Nat. Mater., 2014, 13, 139-150.

101. S. B. Glybovski, S. A. Tretyakov, P. A. Belov, Y. S. Kivshar and C. R. Simovski, Phys. Rep., 2016, 634, 1-72.

102. A. I. Kuznetsov, A. E. Miroshnichenko, M. L. Brongersma, Y. S. Kivshar and B. Luk'yanchuk, Science, 2016, 354, aag2472. 
103. S. Sun, Q. He, J. Hao, S. Xiao and L. Zhou, Adv. Opt. Photon., 2019, 11, 380-479.

104. G. Y. Lee, G. Yoon, S. Y. Lee, H. Yun, J. Cho, K. Lee, H. Kim, J. Rho and B. Lee, Nanoscale, 2018, 10, 4237-4245.

105. S. Chen, Z. Li, Y. Zhang, H. Cheng and J. Tian, Adv. Opt. Mater, 2018, 6, 1800104.

106. J. P. Balthasar Mueller, N. A. Rubin, R. C. Devlin, B. Groever and F, Capasso, Phys. Rev. Lett., 2017, 118, 113901.

107. Z. Li, T. Zhang, Y. Wang, W. Kong, J. Zhang, Y. Huang, C. Wang, X. Li, M. Pu and X. Luo, Laser Photonics Rev., 2018, 12, 1800064.

108. A. Tittl, A. Leitis, M. Liu, F. Yesilkoy, D. Y. Choi, D. N. Neshev, Y. S. Kivshar and H. Altug, Science, 2018, 360, 1105.

109. J. Li, S. Kamin, G. Zheng, F. Neubrech, S. Zhang and N. Liu, Sci. Adv, 2018, 4, eaar6768.

110. C. C. Chang, W. J. M. Kort-Kamp, J. Nogan, T. S. Luk, A. K. Azad, A. J. Taylor, D. A. R. Dalvit, M. Sykora and H. T. Chen, Nano Lett., 2018, 18, 7665-7673.

111. H. T. Miyazaki, T. Kasaya, M. Iwanaga, B. Choi, Y. Sugimoto and K. Sakoda, Appl. Phys. Lett., 2014, 105, 121107.

112. D. Costantini, A. Lefebvre, A. L. Coutrot, I. Moldovan-Doyen, J. P. Hugonin, S. Boutami, F. Marquier, H. Benisty and J. J. Greffet, Phys. Rev. Appl., 2015, 4, 014023.

113. S. Butun and K. Aydin, Opt. Express, 2014, 22, 19457-19468.

114. C. Zhang, J. Yang, W. Yuan, J. Zhao, J. Y. Dai, T. C. Guo, J. Liang, G. Y. Xu, Q. Cheng and T. J. Cui, J. Phys. D: Appl. Phys., 2017, 50, 444002.

115. F. Ding, A. Pors and S. I. Bozhevolnyi, Rep. Prog. Phys., 2017, 81, 026401.

116. C. Pfeiffer, N. K. Emani, A. M. Shaltout, A. Boltasseva, V. M. Shalaev and A. Grbic, Nano Lett., 2014, 14, 2491-2497.

117. J. Liu, Z. Li, W. Liu, H. Cheng, S. Chen and J. Tian, Adv. Opt. Mater, 2016, 4, 2028-2034.

118. Z. Li, W. Liu, H. Cheng, J. Liu, S. Chen and J. Tian, Sci. Rep-UK, 2016, 6, 35485.

119. Z. Y. Wang, R. J. Zhang, S. Y. Wang, M. Lu, X. Chen, Y. X. Zheng, L. Y. Chen, Z. Ye, C. Z. Wang and K. M. Ho, Sci. Rep-UK, 2015, 5, 7810.

120. N. Odebo Länk, R. Verre, P. Johansson and M. Käll, Nano Lett., 2017, 17, 3054-3060.

121. H. Fredriksson, Y. Alaverdyan, A. Dmitriev, C. Langhammer, D. S. Sutherland, M. Zäch and B. Kasemo, Adv. Mater., 2007, 19, 4297-4302.

122. C. Y. Yang, J. H. Yang, Z. Y. Yang, Z. X. Zhou, M. G. Sun, V. E. Babicheva and K. P. Chen, ACS Photonics, 2018, 5, 2596-2601.

123. H. Chalabi, A. Alù and M. L. Brongersma, Phys. Rev. B, 2016, 94, 094307.

124. N. Dahan, Y. Gorodetski, K. Frischwasser, V. Kleiner and E. Hasman, Phys. Rev. Lett., 2010, 105, 136402.

125. D. Lin, P. Fan, E. Hasman and M. L. Brongersma, Science, 2014, 345, 298.

126. A. Kazemi Moridani, R. Zando, W. Xie, I. Howell, J. J. Watkins and J. H. Lee, Adv. Opt. Mater., 2017, 5, 1600993.

127. Y. Xiao, N. A. Charipar, J. Salman, A. Piqué and M. A. Kats, Light-Sci. Appl., 2019, 8, 51.

128. Y. Qu, Q. Li, K. Du, L. Cai, J. Lu and M. Qiu, Laser Photonics Rev., 2017, 11, 1700091.

129. Q. He, S. Sun and L. Zhou, Research, 2019, 2019, 16.

130. R. Sun, P. Zhou, W. Ai, Y. Liu, Y. Li, R. Jiang, W. Li, X. Weng, L. Bi and L. Deng, Opt. Express, 2019, 27, 11537-11546.

131. T. Cao, X. Zhang, W. Dong, L. Lu, X. Zhou, X. Zhuang, J. Deng, X. Cheng, G. Li and R. E. Simpson, Adv. Opt. Mater., 2018, 6, 1800169.

132. S. Vassant, I. Moldovan Doyen, F. Marquier, F. Pardo, U. Gennser, A. Cavanna, J. L. Pelouard and J. J. Greffet, Appl. Phys. Lett., 2013, 102, 081125 .

133. D. Wasserman, Light-Sci. Appl., 2019, 8, 68.

134. J. Park, J. H. Kang, X. Liu, S. J. Maddox, K. Tang, P. C. McIntyre, S. R. Bank and M. L. Brongersma, Sci. Adv., 2018, 4, eaat3163.

135. X. Liu and W. J. Padilla, Optica, 2017, 4, 430-433.

136. S. N. Burokur, J. P. Daniel, P. Ratajczak and A. de Lustrac, Appl. Phys. Lett., 2010, 97, 064101.

137. Y. C. Jun, J. Reno, T. Ribaudo, E. Shaner, J. J. Greffet, S. Vassant, F. Marquier, M. Sinclair and I. Brener, Nano Lett., 2013, 13, 5391-5396.

138. D. Li and C. Z. Ning, Opt. Express, 2011, 19, 14594-14603.

139. P. C. Wu, R. A. Pala, G. Kafaie Shirmanesh, W. H. Cheng, R. Sokhoyan,
M. Grajower, M. Z. Alam, D. Lee and H. A. Atwater, Nat. Commun., 2019, 10, 3654 .

140. D. Shrekenhamer, W. C. Chen and W. J. Padilla, Phys. Rev. Lett., 2013, 110, 177403

141. T. Inoue, M. D. Zoysa, T. Asano and S. Noda, Nat. Mater, 2014, 13, 928.

142. S. Inampudi and H. Mosallaei, Phys. Rev. B, 2017, 96, 125407.

143. Z. Y. Yang, S. Ishii, T. Yokoyama, T. D. Dao, M. G. Sun, P. S. Pankin, I. V Timofeev, T. Nagao and K. P. Chen, ACS Photonics, 2017, 4, 2212-2219.

144. H. Zhu, H. Luo, Q. Li, D. Zhao, L. Cai, K. Du, Z. Xu, P. Ghosh and M. Qiu, Opt. Lett., 2018, 43, 5230-5233.

145. M. De Zoysa, T. Asano, K. Mochizuki, A. Oskooi, T. Inoue and S. Noda, Nat. Photonics, 2012, 6, 535 .

146. T. Inoue, T. Asano, M. De Zoysa, A. Oskooi and S. Noda, J. Opt. Soc. Am. $B, 2013,30,165-172$.

147. T. Inoue, M. De Zoysa, T. Asano and S. Noda, Appl. Phys. Lett., 2013, 102, 191110.

148. T. Inoue, M. De Zoysa, T. Asano and S. Noda, Phys. Rev. B, 2015, 91 235316.

149. G. Biener, N. Dahan, A. Niv, V. Kleiner and E. Hasman, Appl. Phys. Lett., 2008, 92, 081913

150. Y. Guo and S. Fan, Opt. Express, 2016, 24, 29896-29907.

151. M. O. Ali, N. Tait and S. Gupta, J. Opt. Soc. Am. A, 2018, 35, 119-124.

152. N. Shitrit, I. Yulevich, E. Maguid, D. Ozeri, D. Veksler, V. Kleiner and E. Hasman, Science, 2013, 340, 724.

153. S. A. Dyakov, V. A. Semenenko, N. A. Gippius and S. G. Tikhodeev, Phys Rev. B, 2018, 98, 235416.

154. K. Ikeda, H. T. Miyazaki, T. Kasaya, K. Yamamoto, Y. Inoue, K. Fujimura T. Kanakugi, M. Okada, K. Hatade and S. Kitagawa, Appl. Phys. Lett., 2008, 92, 021117.

155. S. L. Wadsworth, P. G. Clem, E. D. Branson and G. D. Boreman, Opt Mater. Express, 2011, 1, 466-479.

156. M. Song, H. Yu, J. Luo and Z. Zhang, Plasmonics, 2017, 12, 649-654.

157. O. G. Kollyukh, A. I. Liptuga, V. Morozhenko and V. I. Pipa, Phys. Rev. B, 2005, 71, 073306.

158. J. C. W. Lee and C. T. Chan, Appl. Phys. Lett., 2007, 90, 051912.

159. C. Wu, N. Arju, G. Kelp, J. A. Fan, J. Dominguez, E. Gonzales, E. Tutuc, I. Brener and G. Shvets, Nat. Commun., 2014, 5, 3892.

160. N. Dahan, A. Niv, G. Biener, V. Kleiner and E. Hasman, Appl. Phys. Lett., 2005, 86, 191102

161. C. Khandekar and Z. Jacob, Phys. Rev. Appl., 2019, 12, 014053.

162. G. Barbillon, E. Sakat, J. P. Hugonin, S. A. Biehs and P. Ben-Abdallah, Opt Express, 2017, 25, 23356-23363.

163. S. Fan and A. Raman, Natl. Sci. Rev., 2018, 5, 132-133.

164. D. Zhao, A. Aili, Y. Zhai, S. Xu, G. Tan, X. Yin and R. Yang, Appl. Phys. $R$ B. Ko, D. Lee, T. Badloe and J. Rho, Energies, 2018, 12

166. M. M. Hossain, B. Jia and M. Gu, Adv. Opt. Mater., 2015, 3, 1047-1051.

167. C. Zou, G. Ren, M. M. Hossain, S. Nirantar, W. Withayachumnankul, T. Ahmed, M. Bhaskaran, S. Sriram, M. Gu and C. Fumeaux, Adv. Opt. Mater., 2017, 5, 1700460.

168. L. Zhu, A. P. Raman and S. Fan, P. Natl. Acad. Sci., 2015, 112, 12282

169. H. Bao, C. Yan, B. Wang, X. Fang, C. Y. Zhao and X. Ruan, Sol. Energ. Mat. Sol. C., 2017, 168, 78-84.

170. A. P. Raman, M. A. Anoma, L. Zhu, E. Rephaeli and S. Fan, Nature, 2014, 515,540 .

171. Y. Cui, K. H. Fung, J. Xu, H. Ma, Y. Jin, S. He and N. X. Fang, Nano Lett., 2012, 12, 1443-1447.

172. B. Zhao and Z. M. Zhang, Nanosc. Microsc. Therm., 2017, 21, 123-133.

173. D. Zhao, A. Aili, Y. Zhai, J. Lu, D. Kidd, G. Tan, X. Yin and R. Yang, Joule, 2019, 3, 111-123.

174. S. Atiganyanun, J. B. Plumley, S. J. Han, K. Hsu, J. Cytrynbaum, T. L. Peng, S. M. Han and S. E. Han, ACS Photonics, 2018, 5, 1181-1187.

175. J. 1. Kou, Z. Jurado, Z. Chen, S. Fan and A. J. Minnich, ACS Photonics, 2017, 4, 626-630.

176. P. Yang, C. Chen and Z. M. Zhang, Sol. Energy, 2018, 169, 316-324.

177. Y. Yang, L. Long, S. Meng, N. Denisuk, L. Wang and Y. Zhu, eprint arXiv:1908.01574, 2019, arXiv:1908.01574.

178. A. Datas and C. Algora, Progress in Photovoltaics: Research and Applications, 2013, 21, 1025-1039. 
179. P. Nagpal, S. E. Han, A. Stein and D. J. Norris, Nano Lett., 2008, 8, 32383243.

180. V. Rinnerbauer, A. Lenert, D. M. Bierman, Y. X. Yeng, W. R. Chan, R. D. Geil, J. J. Senkevich, J. D. Joannopoulos, E. N. Wang, M. Soljačić and I. Celanovic, Adv. Energy Mater., 2014, 4, 1400334.

181. D. N. Woolf, E. A. Kadlec, D. Bethke, A. D. Grine, J. J. Nogan, J. G. Cederberg, D. Bruce Burckel, T. S. Luk, E. A. Shaner and J. M. Hensley, Optica, 2018, 5, 213-218.

182. M. Suemitsu, T. Asano, T. Inoue and S. Noda, ACS Photonics, 2019.

183. R. Wang, J. Shang and J. Huang, Int. J. Therm. Sci., 2018, 131, 14-19.

184. T. Z. Yang, Y. Su, W. Xu and X. D. Yang, Appl. Phys. Lett., 2016, 109, 121905.

185. Y. Li, X. Shen, Z. Wu, J. Huang, Y. Chen, Y. Ni and J. Huang, Phys. Rev. Lett., 2015, 115, 195503.

186. Y. Li, X. Bai, T. Yang, H. Luo and C. W. Qiu, Nat. Commun., 2018, 9, 273.

187. O. Salihoglu, H. B. Uzlu, O. Yakar, S. Aas, O. Balci, N. Kakenov, S. Balci, S. Olcum, S. Süzer and C. Kocabas, Nano Lett., 2018, 18, 4541-4548.

188. L. Xiao, H. Ma, J. Liu, W. Zhao, Y. Jia, Q. Zhao, K. Liu, Y. Wu, Y. Wei, S. Fan and K. Jiang, Nano Lett., 2015, 15, 8365-8370.

189. T. Ozawa, H. M. Price, A. Amo, N. Goldman, M. Hafezi, L. Lu, M. C. Rechtsman, D. Schuster, J. Simon, O. Zilberberg and I. Carusotto, Rev. Mod. Phys., 2019, 91, 015006.
190. R. El-Ganainy, K. G. Makris, M. Khajavikhan, Z. H. Musslimani, S. Rotter and D. N. Christodoulides, Nat. Phys., 2018, 14, 11.

191. D. E. Chang, J. S. Douglas, A. González-Tudela, C. L. Hung and H. J. Kimble, Rev. Mod. Phys., 2018, 90, 031002.

192. M. G. Silveirinha, Phys. Rev. X, 2019, 9, 011037.

193. C. F. Doiron and G. V. Naik, Adv. Mater, 2019, 0, 1904154.

194. A. Ridolfo, S. Savasta and M. J. Hartmann, Phys. Rev. Lett., 2013, 110, 163601.

195. K. F. Mak and J. Shan, Nat. Photonics, 2016, 10, 216.

196. Z. Guo, H. Zhang, S. Lu, Z. Wang, S. Tang, J. Shao, Z. Sun, H. Xie, H. Wang, X. F. Yu and P. K. Chu, Adv. Funct. Mater, 2015, 25, 6996-7002.

197. K. Watanabe, T. Taniguchi, T. Niiyama, K. Miya and M. Taniguchi, Nat. Photonics, 2009, 3, 591-594.

198. J. C. W. Song and N. M. Gabor, Nat. Nanotechnol., 2018, 13, 986-993.

199. X. L. Qi and S. C. Zhang, Rev. Mod. Phys., 2011, 83, 1057-1110.

200. R. J. Shiue, Y. Gao, C. Tan, C. Peng, J. Zheng, D. K. Efetov, Y. D. Kim, J. Hone and D. Englund, Nat. Commun., 2019, 10, 109.

201. M. Xie, S. Zhang, B. Cai, Y. Gu, X. Liu, E. Kan and H. Zeng, Nano Energy, 2017, 38, 561-568

Publisher's Note Engineered Science Publisher remains neutral with regard to jurisdictional claims in published maps and institutional affiliations. 